\title{
Comparison of the Effectiveness of Sertraline, Transcranial Direct Stimulation Current and their Combination on Post-Traumatic Stress Disorder in Veterans
}

\section{Shabnam Sarhadi ${ }^{1}$, Fatemeh Ghaemi ${ }^{2 *}$, Fariborz Dortaj ${ }^{3}$, Ali Delavar ${ }^{3}$}

${ }^{1}$ Department of Psychology, Factuly of Literature, Humanities and Social Science, Science and Research Branch, Islamic Azad University, Tehran, Iran

${ }^{2}$ Ministry of Health and Medical Education, Deputy of Health, Tehran, Iran

${ }^{3}$ Department of Educational Psychology, Faculty of Psychology and Educational Sciences, Allameh Tabataba'i University, Tehran, Iran

\section{Article Info:}

\section{ABSTRACT}

Introduction: Traumatic Stress Disorder (PTSD) is one of the most prevalent health problems in the victims of the Iran-Iraq war. Despite many advantages of the administration of selective serotonin reuptake inhibitors, as the first-line treatment of PTSD, a large number of the patients still need more effective therapies. The purpose of this study was to comparison of the effectiveness of sertraline, transcranial direct stimulation current (TDCS), and the combined treatment (TDCS and sertraline) to relieve the symptoms of PTSD in the veterans in Tehran, Iran. Materials and Methods: The current study was a semi-experimental study including pre- and post-tests, a control group, and three-month follow-up. The population of study was veterans who lived in Tehran in 2018. 68 veterans were selected by purposive sampling method and then randomly divided to control and experimental groups. The firstgroup was treated with sertraline, the second group received TDCS, and the third group treated with the combined treatment. The PTSD Checklist for DSM-5(PCL-5) was used as a research tool. Results: All of the treatment approaches were effective in reduction of symptoms in patients with PTSD. In the post-test and follow-up phase, there was a significant difference in PCL-5 scores between sertraline, TDCS, and combined treatment in comparison with the control group. Combined treatment (TDCS and sertraline) have shown a significantly higher and persistence effectiveness. Conclusion: Combined treatment with TDCS and sertraline can be used as an efficient and effective approach to reduce PTSD symptoms in veterans.

Key words:

1. Transcranial Direct

Current Stimulation

2. Sertraline

3. Veterans

4. Combat Disorders

*Corresponding Author: Fatemeh Ghaemi

E-mail: ghaemifatemeh@yahoo.com 


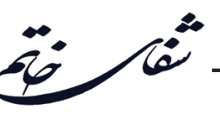

مقايسة اثربخشى سر ترالين، تحريك جريان مستقيم فراجمجمهاى و تركيب آنها در اختلال استرس يس از سانحه در جانبازان

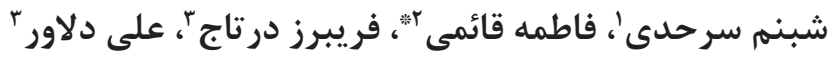 \\ 'كروه روانشناسى، دانشكده ادبيات، علوم انسانى و اجتماعى، واحد علوم و تحقيقات، دانشعاه آزاد اسلامى، تهران، ايران

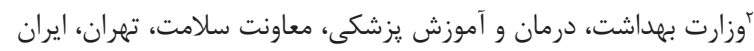

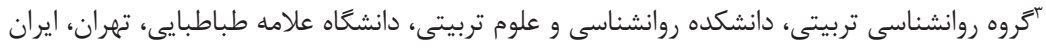

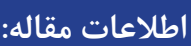

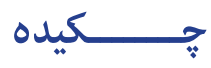

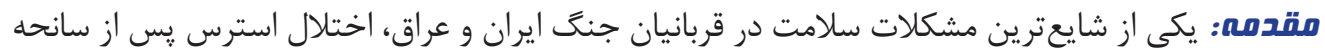

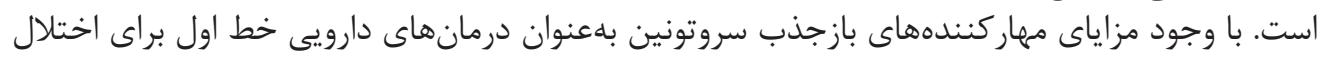

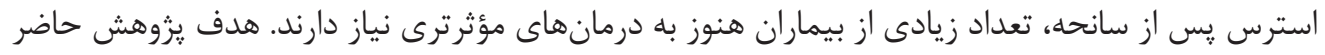

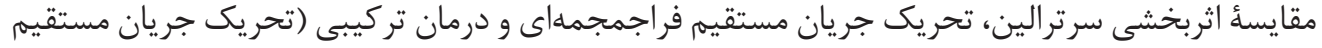

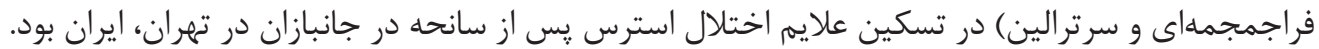

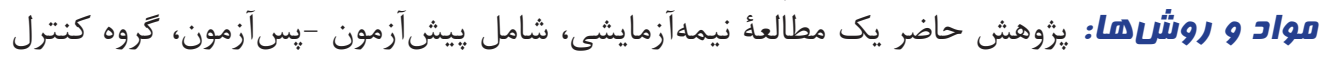

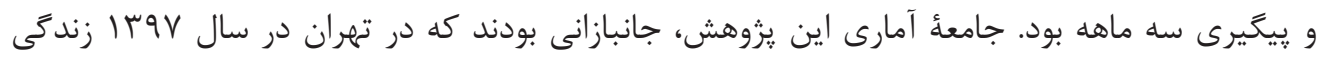

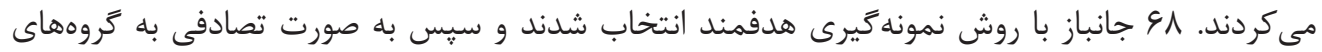

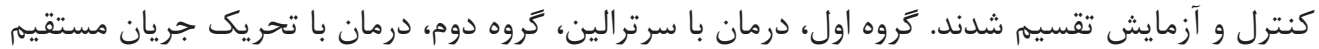

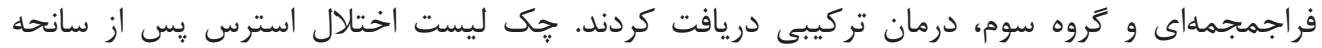

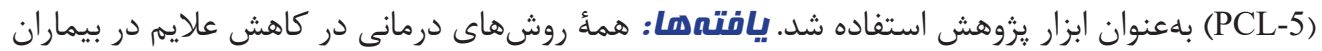

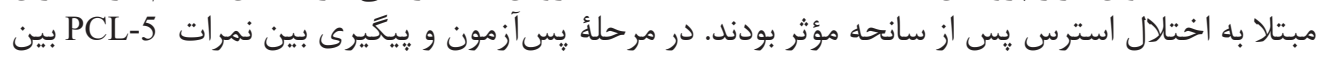

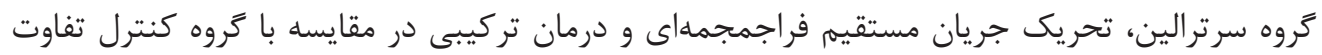

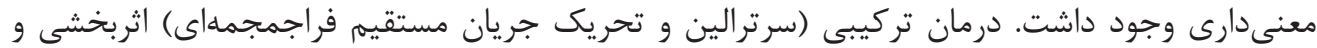

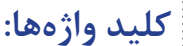

1. (1) تحريك جريان مستقيم

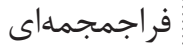

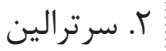

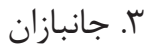

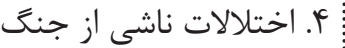

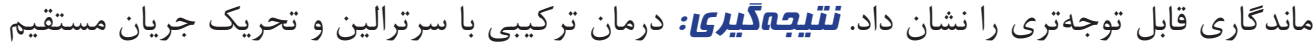

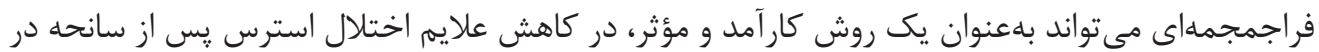
جانبازان مورد استفاده قرار كيرد.
" نويسنده مسئول: فاطمه قائمى

آدرس الكترونيكى: ghaemifatemeh@yahoo.com 
كمتـــر در قشـــر بيش ييشــانى جانبــى قدامــى (VLPFC)

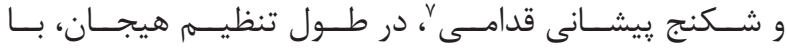

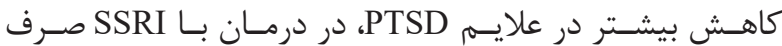

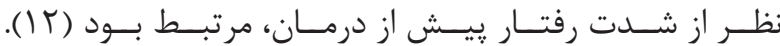
SSRI

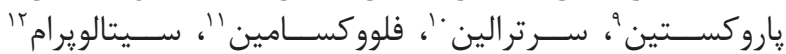

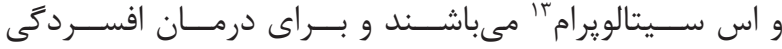

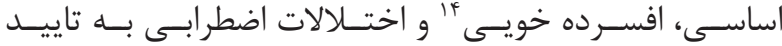

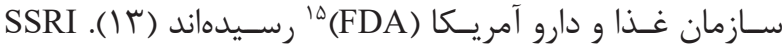

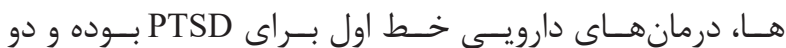

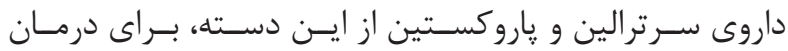

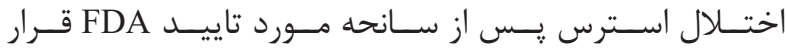

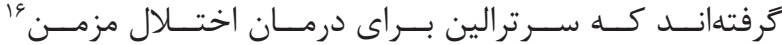

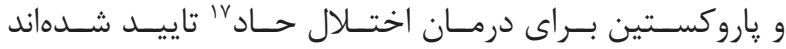

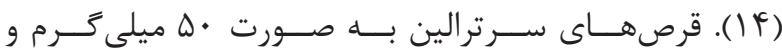

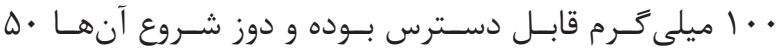

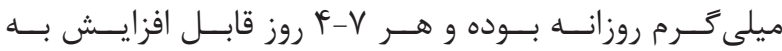

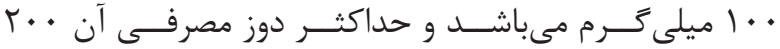

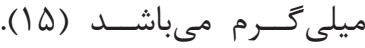
اختـلالات در عملكــــد شـناختى، علايـهم اصلـى در اختــلالات

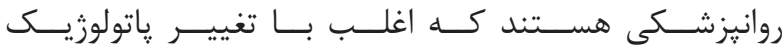

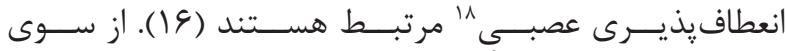

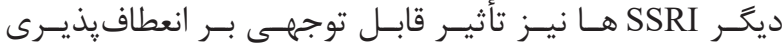

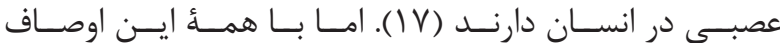

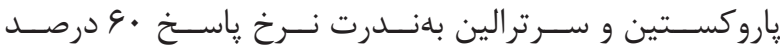

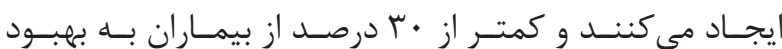

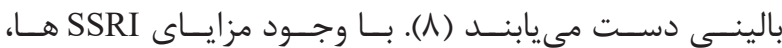

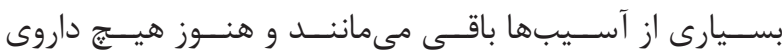

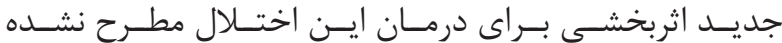

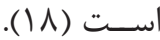

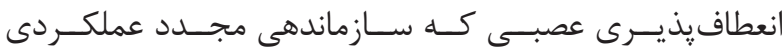

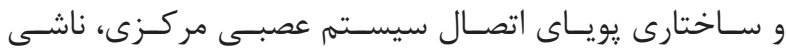

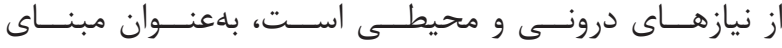

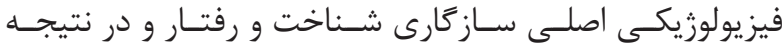

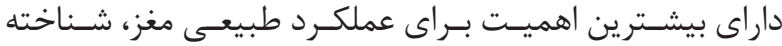

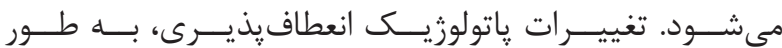

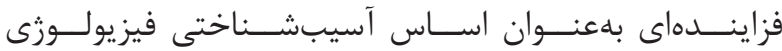

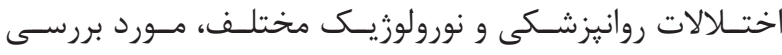

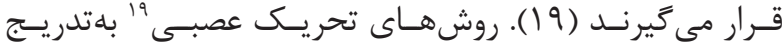

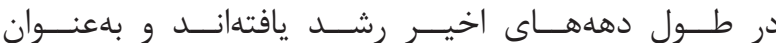

${ }^{1}$ Post-traumatic stress disorder

${ }^{2}$ The diagnostic and statistical manual of mental disorders, fifth edition

${ }^{3}$ Selective serotonin re-uptake inhibitors

${ }^{4}$ Dorsolateral prefrontal cortex

${ }^{5}$ Supplementary motor area

${ }^{6}$ Ventrolateral prefrontal cortex

${ }^{7}$ Inferior frontal gyrus

${ }^{8}$ Fluoxetine

${ }^{9}$ Paroxetine

${ }^{10}$ Sertraline

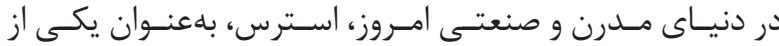

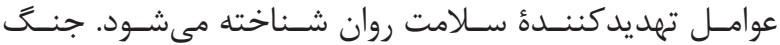

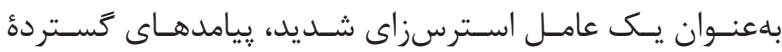

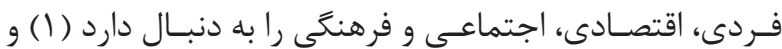

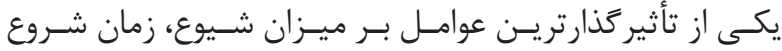

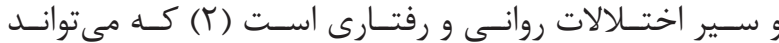

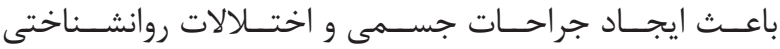

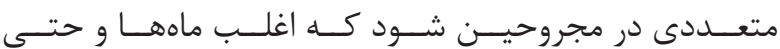

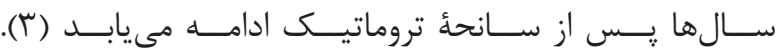

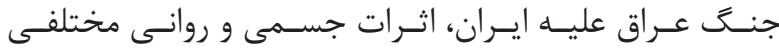

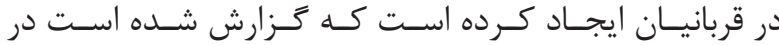

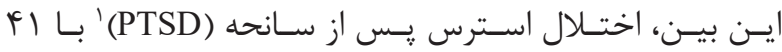

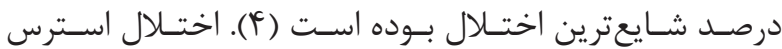

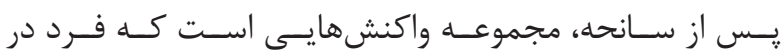

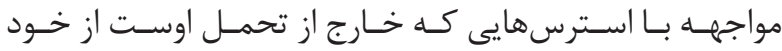

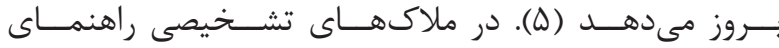

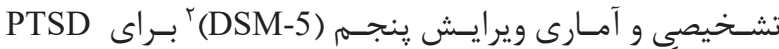

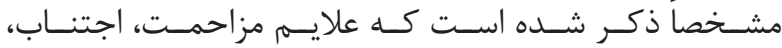

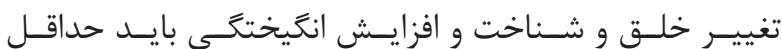

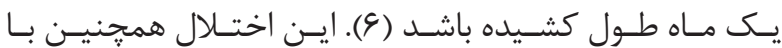

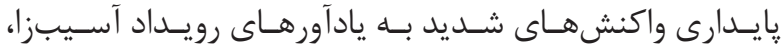

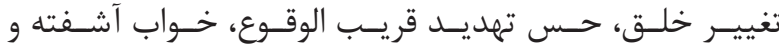

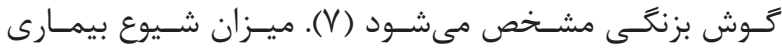

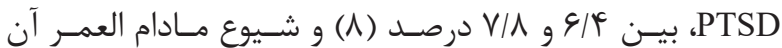

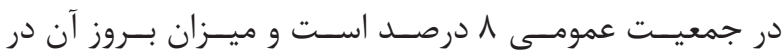

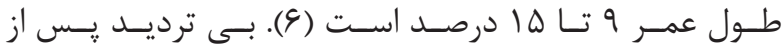

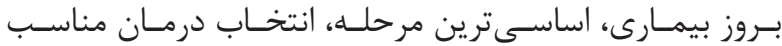

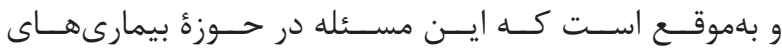

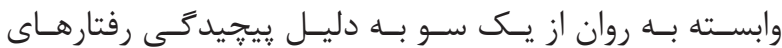

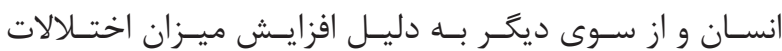

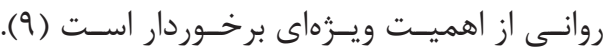

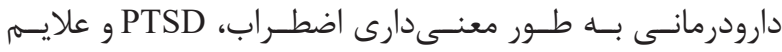

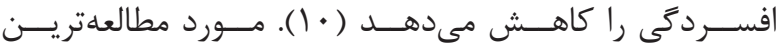

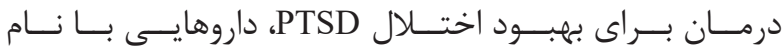

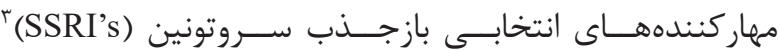

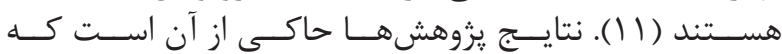

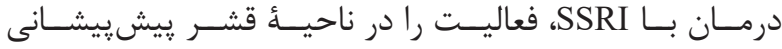

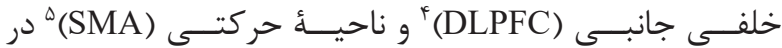

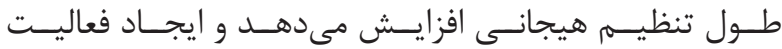

\footnotetext{
${ }^{11}$ Fluvoxamine

${ }^{12}$ Citalopram

${ }^{13}$ Escitalopram

${ }^{14}$ Dysthymia

${ }^{15}$ The food and drug administration

${ }^{16}$ Chronic

${ }^{17}$ Acute

${ }^{18}$ Neuroplasticity

${ }^{19}$ Neural stimulation
} 


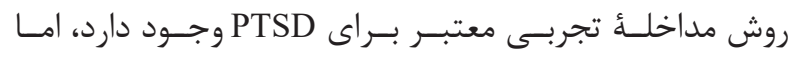

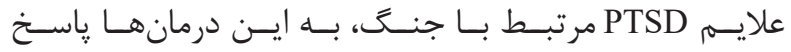

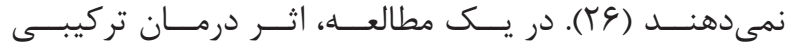

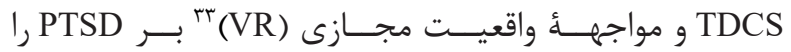

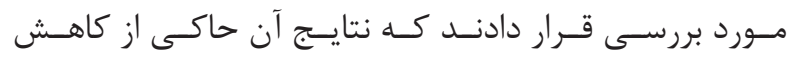

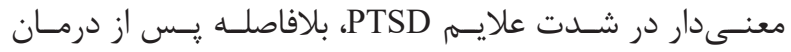

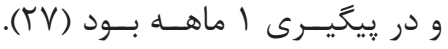

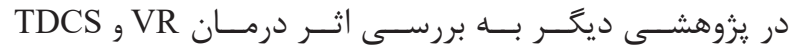

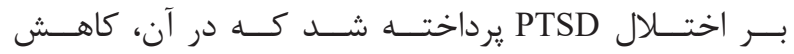

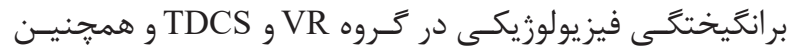

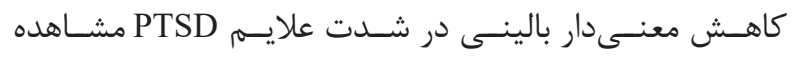

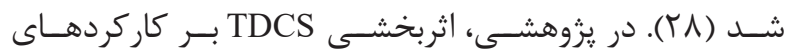

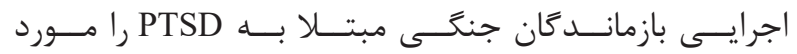

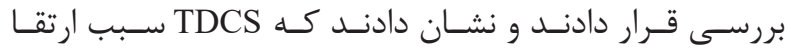

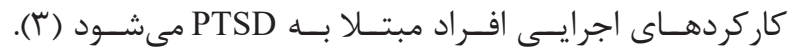

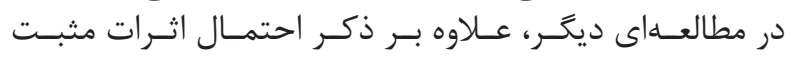

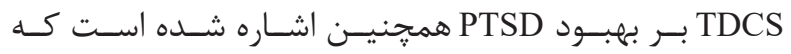

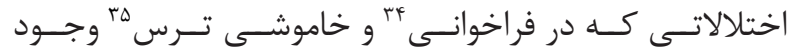

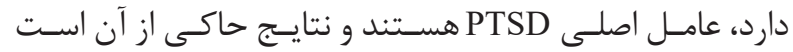

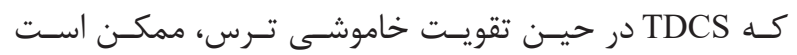

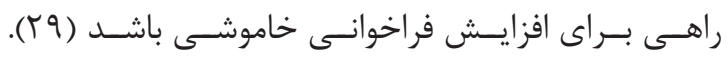

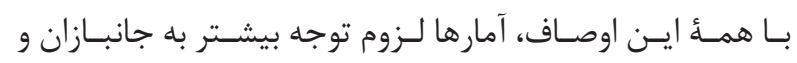

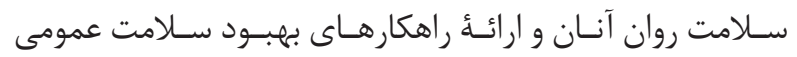

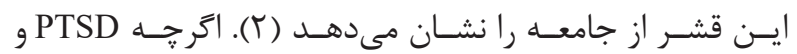

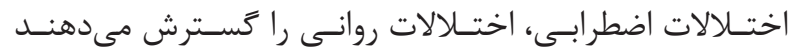

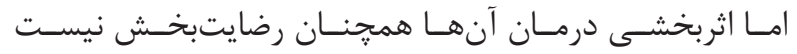

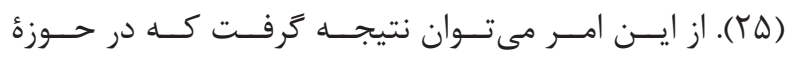

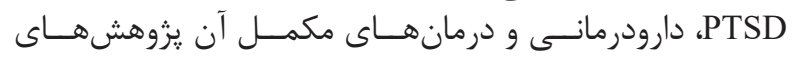

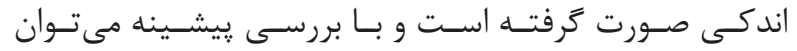

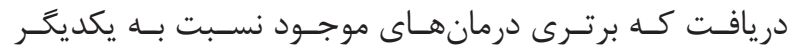

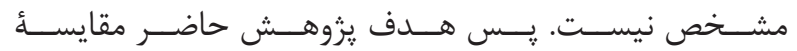

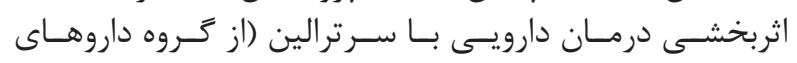

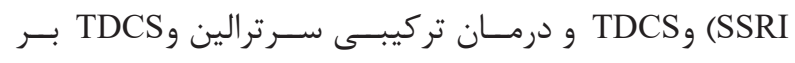

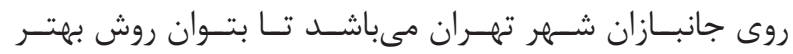

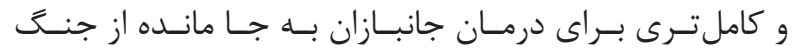

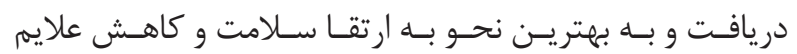

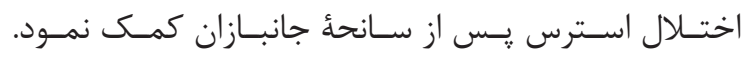

مواد و روشها

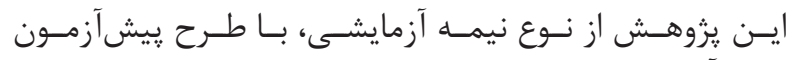

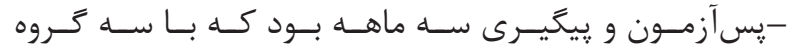

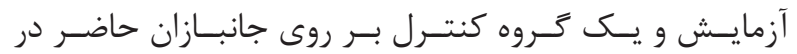

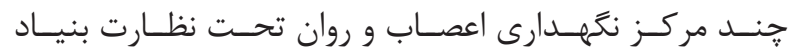

${ }^{20}$ Noninvasive brain stimulations

${ }^{21}$ Repetitive transcranial magnetic stimulation

${ }^{22}$ Transcranial direct current stimulation

${ }^{23}$ Pathologic plasticity

${ }^{24}$ Cortical excitability

${ }^{25}$ Facilitate

${ }^{26}$ Inhibition

${ }^{27}$ Anode

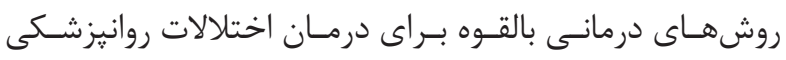

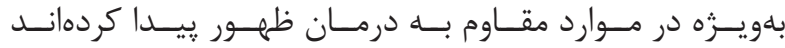

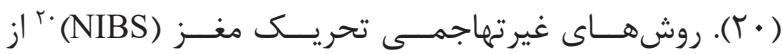

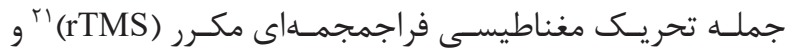

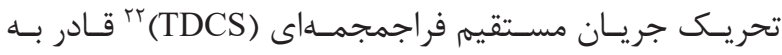

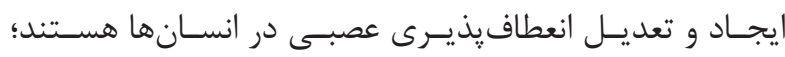

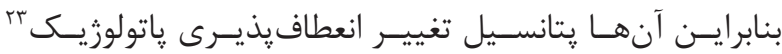

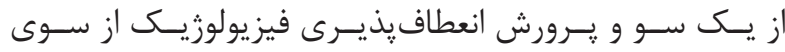

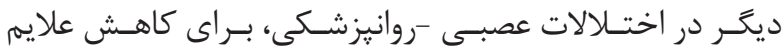

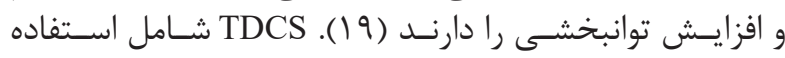

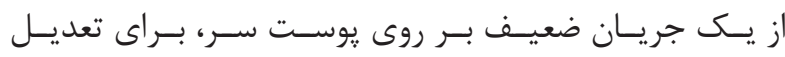

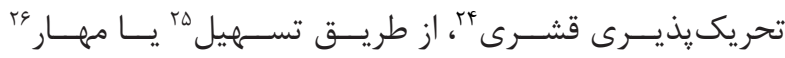

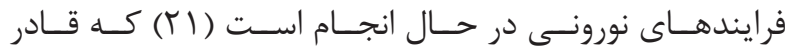

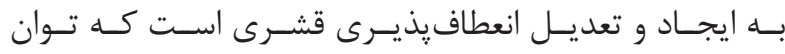

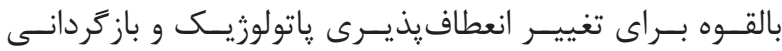

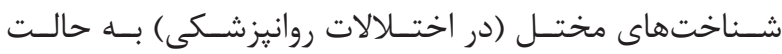
اوليسه را دارد (1) (1).

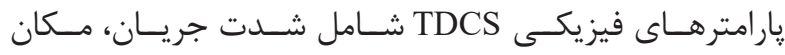

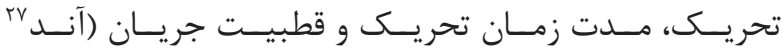

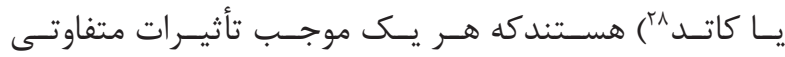

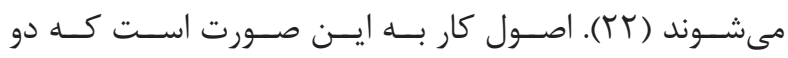

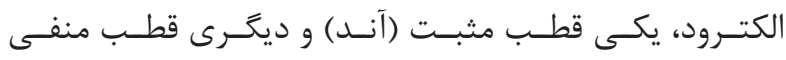

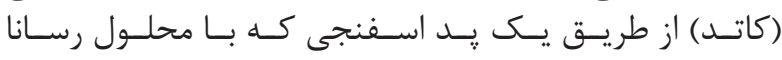

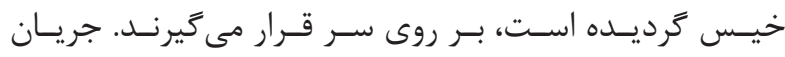

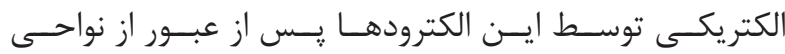

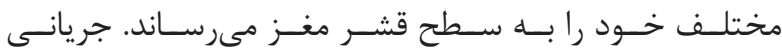

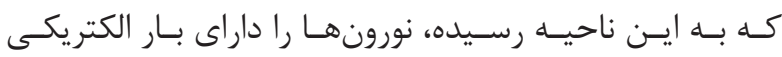

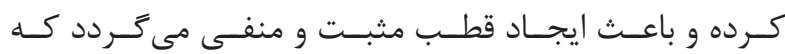

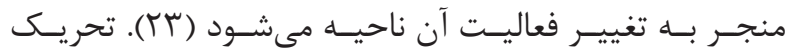

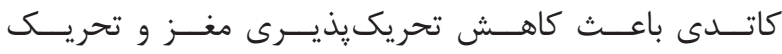

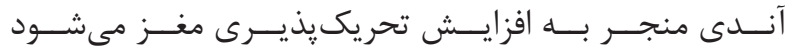
طا (YF)

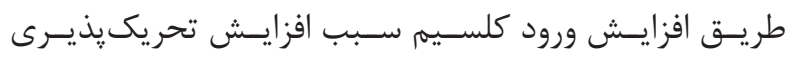
مىشـود (IV) (IV)

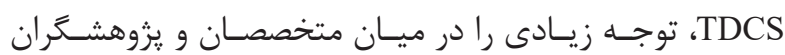

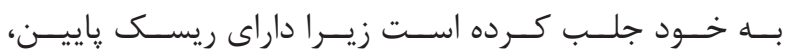

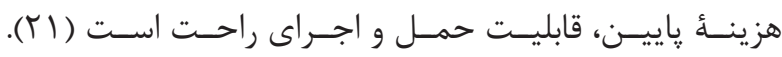

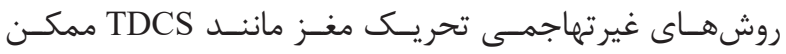

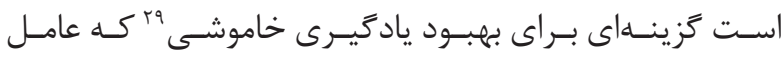

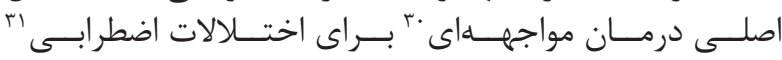

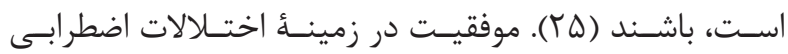

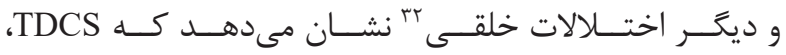

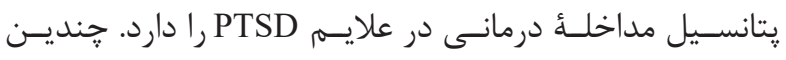

${ }^{28}$ Cathode

${ }^{29}$ Extiction learning

${ }^{30}$ Therapy exposure

${ }^{31}$ Anxiety disorders

${ }^{32}$ Mood disorders

${ }^{33}$ Virtual reality exposure therapy

${ }^{34}$ Recall

${ }^{35}$ Fear extinction 


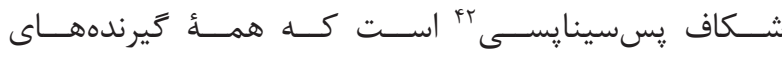

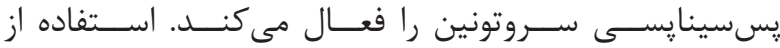

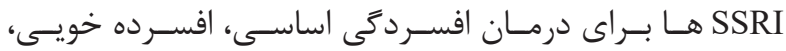

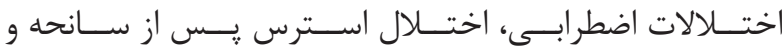

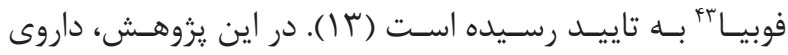

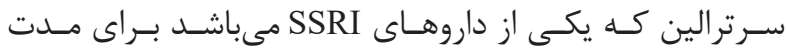

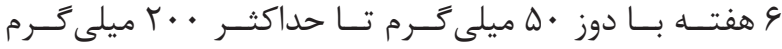

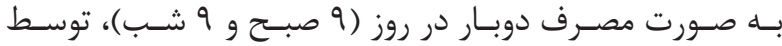

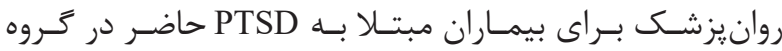

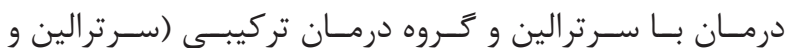
(TDCS

تحريك جريان مستقيم فراجمجمهاى

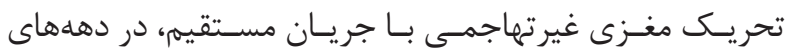

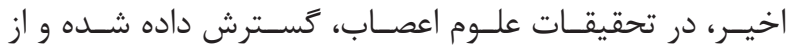

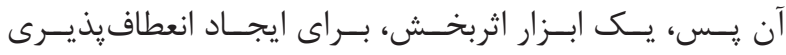

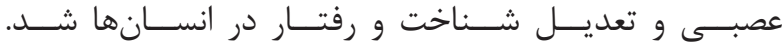

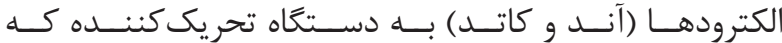

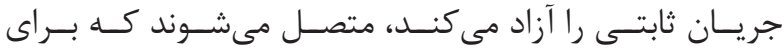

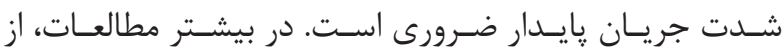

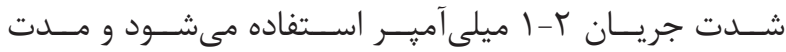

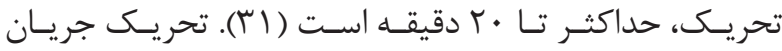

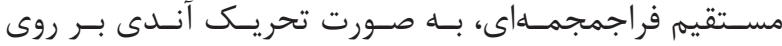

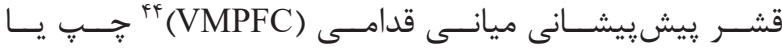

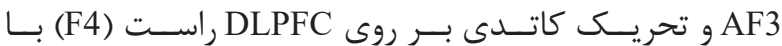

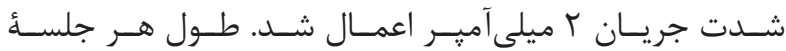

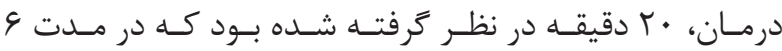

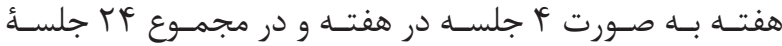

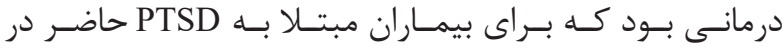

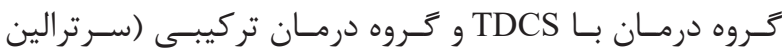

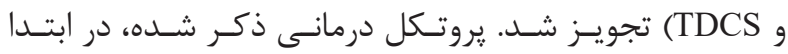

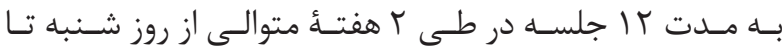

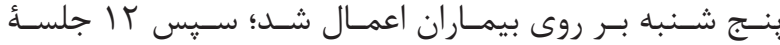

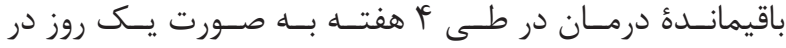

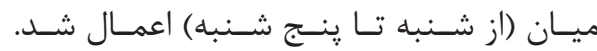

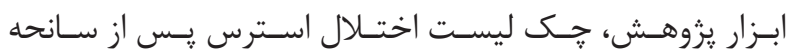

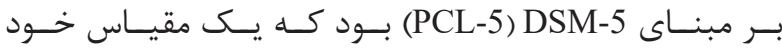

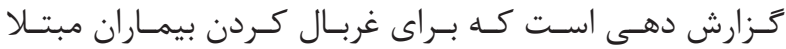

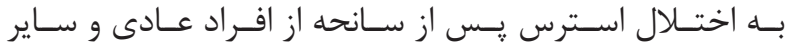

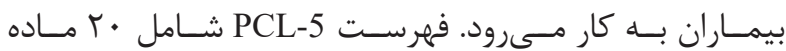

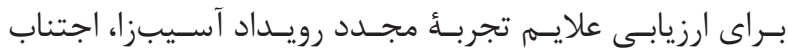

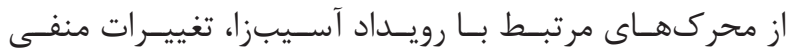

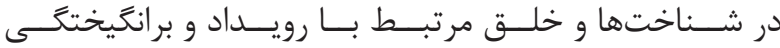

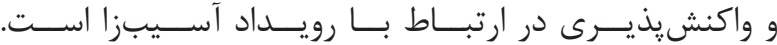

${ }^{36}$ PTSD checklist for DSM-5

${ }^{37}$ Asentra

${ }^{38}$ Zoloft

${ }^{39}$ Lustral

${ }^{40}$ Obsessive-compulsive disorder

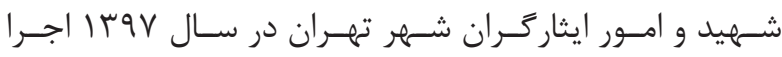

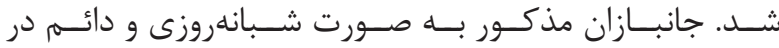

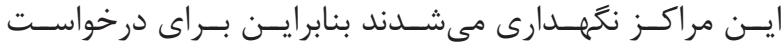

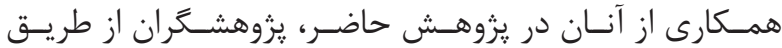

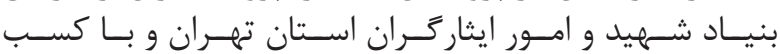

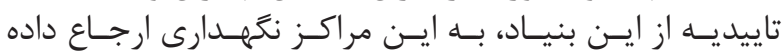

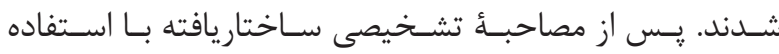

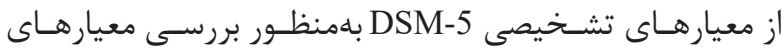

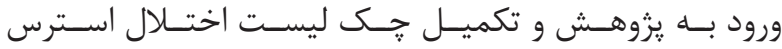

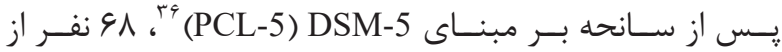

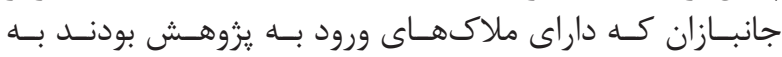

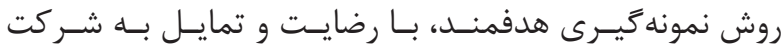

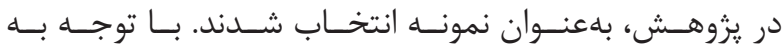

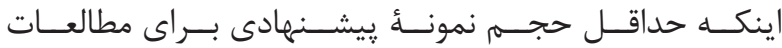

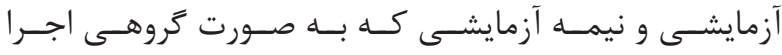

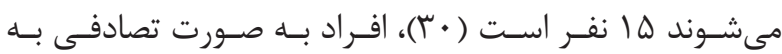

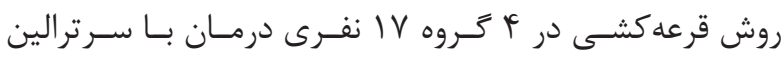

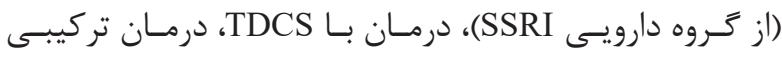

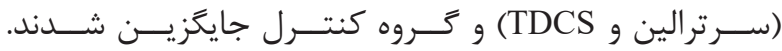

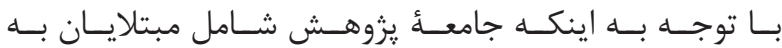

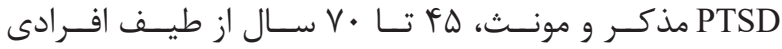

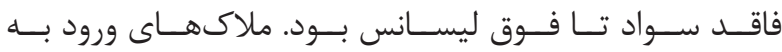

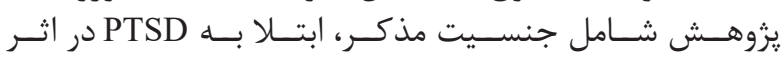

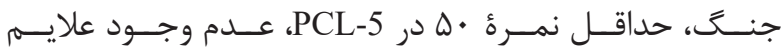

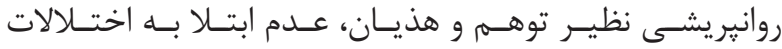

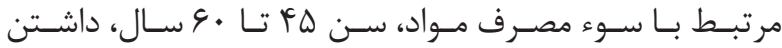

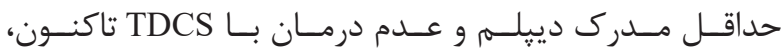

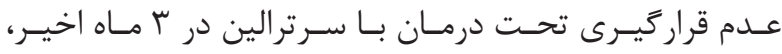

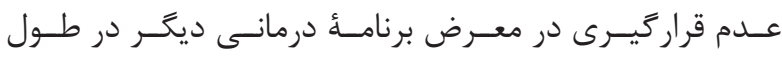

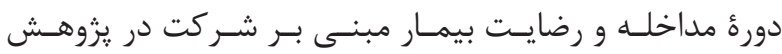

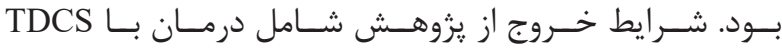

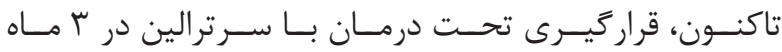

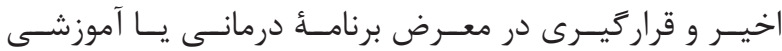

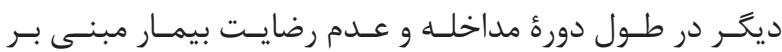

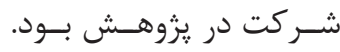

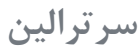

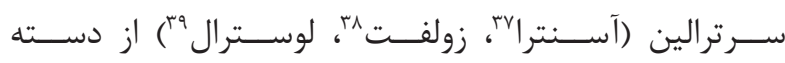

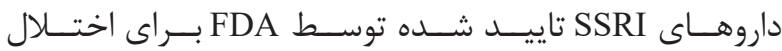

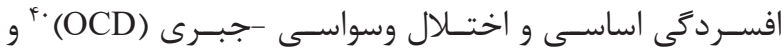

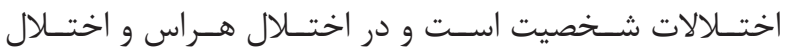

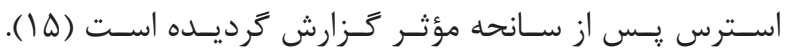

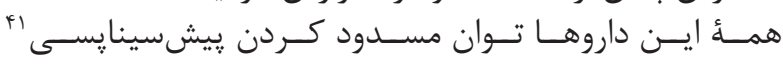

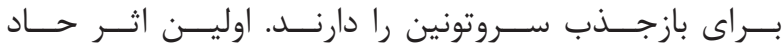

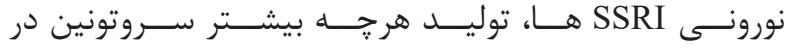

\footnotetext{
${ }^{41}$ Pre-synaptic

${ }^{42}$ Post-synaptic

${ }^{43}$ Phobia

${ }^{44}$ Ventromedial prefrontal cortex
} 


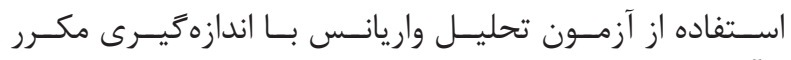

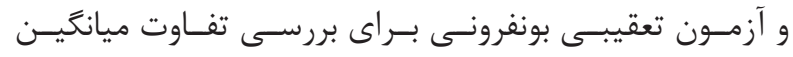

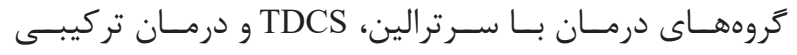

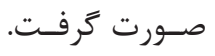

بافتهها

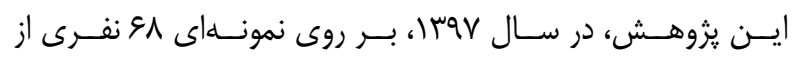

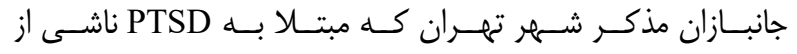

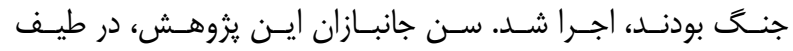

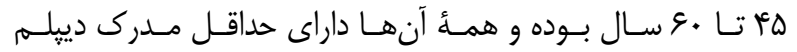

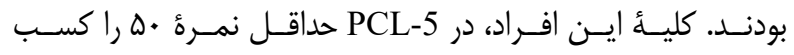

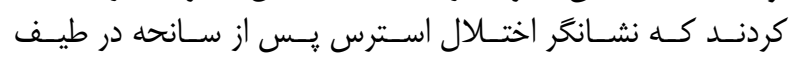

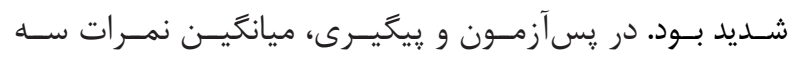

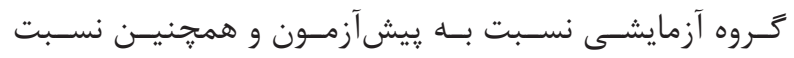

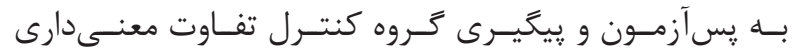

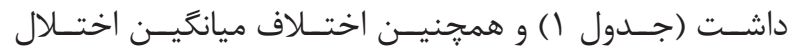

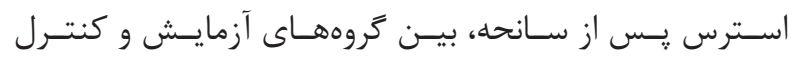

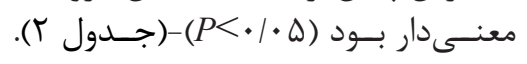

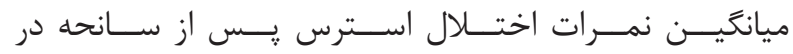

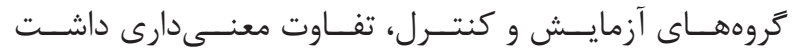

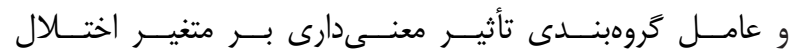

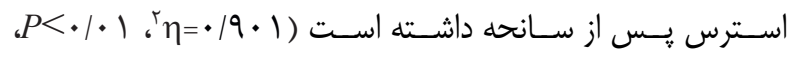

.

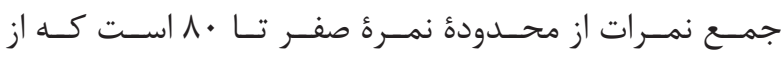

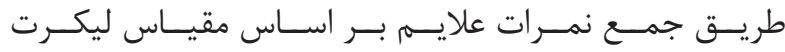

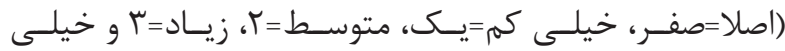

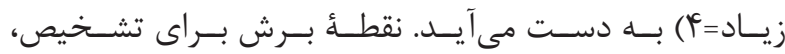

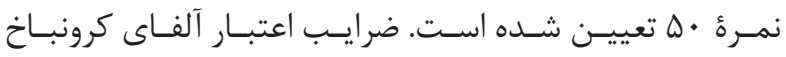

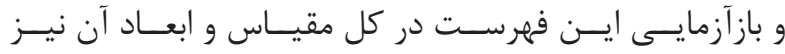

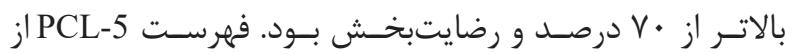

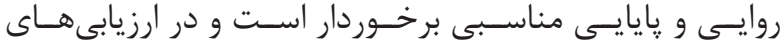

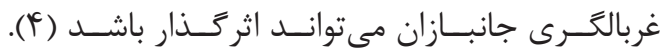

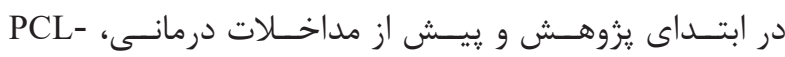

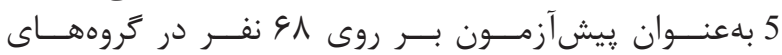

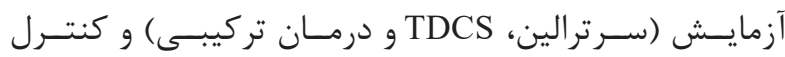

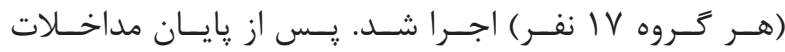

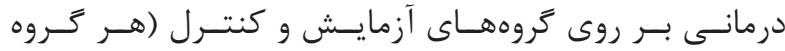

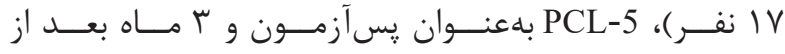

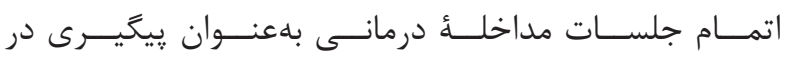

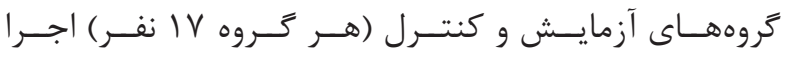

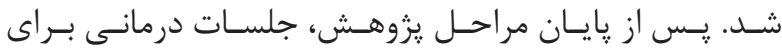

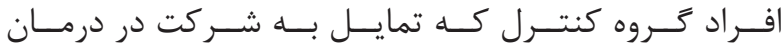

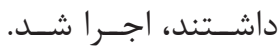
تجزيه و تحليل دادهها

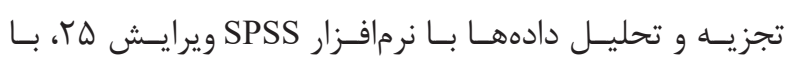

\begin{tabular}{|c|c|c|c|c|c|c|}
\hline اتحراف معيار & ميانكين بيكيرى & انحراف معيار & ميانكين بس آزمون & انحراف معيار & ميانكين بيش آزمون & 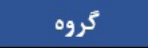 \\
\hline 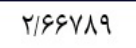 & $\Delta r / q 4 V \mid$ & T/GNIG4 & rG/VGFV & $r / V f \Delta \Delta q$ & $99 / 1 V 90$ & سرترالين \\
\hline T/DVGVG & $\Delta r / \Delta T q F$ & $r / \Delta \& A 19$ & $F V / V \cdot \Delta q$ & $f / r q \ldots f$ & GD/ATHA & TDCS \\
\hline T/DGYFE & rg/Trar & T/GYTFY & rQ/QMAT & r/VAVTL & $99194 \mathrm{VI}$ & درمان تركيبى \\
\hline$r / 99119$ & $99111 \mathrm{VS}$ & $4 / .9111$ & gQ/TQRq & $f / f \cdot \wedge r \wedge$ & $991 \cdot \Delta \Lambda \Lambda$ & كنترل \\
\hline
\end{tabular}

جدول ا- ميانكَين آمارى نمرات اختلال استرس يس از سانحه به تفكيك گروه در سه مرحله در جانبازان (هر گروه VI نفر).

\begin{tabular}{|c|c|c|c|c|}
\hline سطح معنى & تفاوت ميانگين (كروه ا و ؟) & rog & كروه & متغير \\
\hline$\cdot 1 \cdot 1 \Delta$ & $-r / \uparrow q \cdot *$ & TDCS & \multirow{3}{*}{ سرترالين } & \multirow{12}{*}{ اختلال استرس از سانحه } \\
\hline$\cdot 1 \cdots$ & $I T / V \cdot q^{* *}$ & درمان تركيبى & & \\
\hline$\cdot / \cdots$ & $-\mid M / \varphi f V^{* *}$ & كنترل & & \\
\hline$\cdot 1 \cdot 10$ & $r / f q \cdot *$ & سرترالين & \multirow{3}{*}{ TDCS } & \\
\hline$\cdot / \cdots$ & $19 / 194^{* *}$ & درمان تركيبى & & \\
\hline$\cdot 1 \cdots$ & $* *-1 \cdot / 1 \Delta V$ & كنترل & & \\
\hline$\cdot 1 \cdots$ & $-1 Y / V \cdot \varphi^{* *}$ & 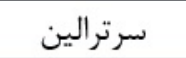 & \multirow{3}{*}{ تر كيبي } & \\
\hline$\cdot 1 \cdots$ & $-19 / 194^{* *}$ & TDCS & & \\
\hline$\cdot / \cdots$ & $-r q / r \Delta \mu^{* *}$ & كنترل & & \\
\hline$\cdot / \cdots$ & $\mid$ T/GFV** & 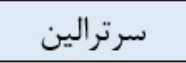 & \multirow{3}{*}{ كنترل } & \\
\hline$\cdot 1 \cdots$ & $* * 1 \cdot / 1 \Delta V$ & TDCS & & \\
\hline$\cdot 1 \cdots$ & rg/ruH** & درمان تركيبى & & \\
\hline
\end{tabular}

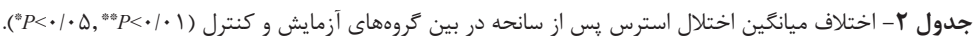


جدول بـ- نتايج آزمون اثر بين آزمودنى (كرومبندى) در متغير اختلال استرس يس از سانحة جانبازان.

\begin{tabular}{|c|c|c|c|c|c|}
\hline اندازة اثر & سطح معنىدارى & مقدار F & درجهُ آزادى & منبع تغييرات & متغير \\
\hline $9 \cdot 1 /$. & $\ldots /$ & $11 . / 194$ & r & تروه & اختلال استرس \\
\hline- & - & - & gF & خطا & يس از سانحه \\
\hline
\end{tabular}

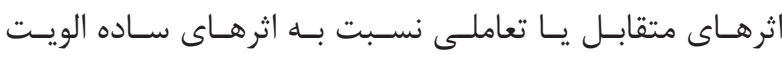

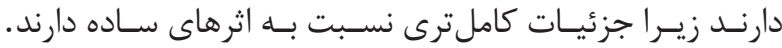

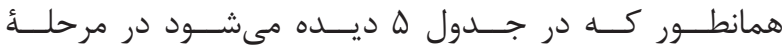

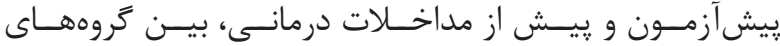

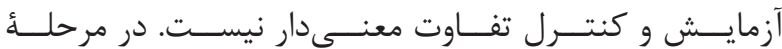

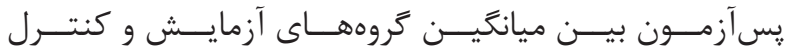

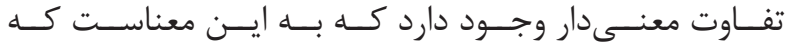

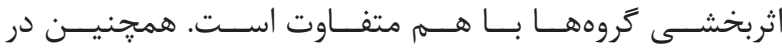

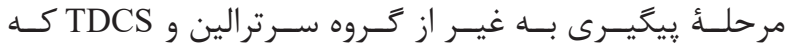

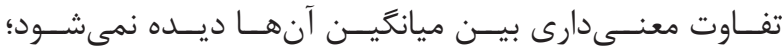

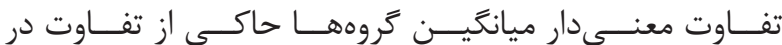

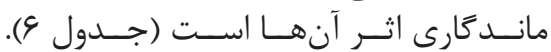

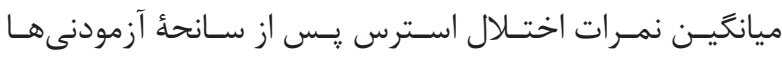

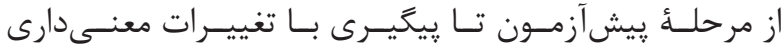

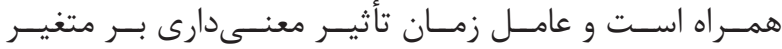

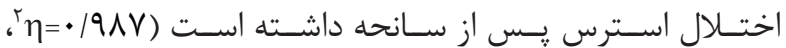

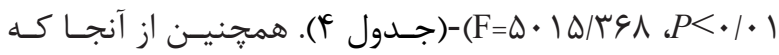

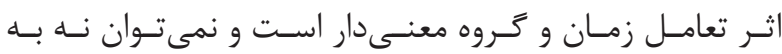

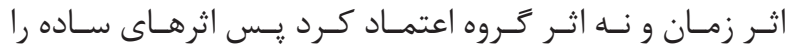

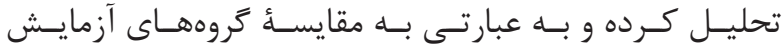

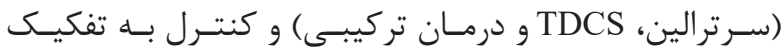

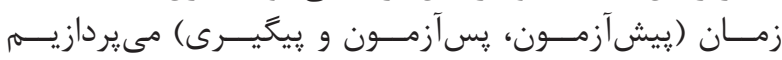

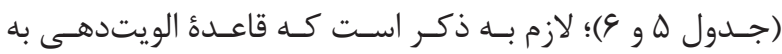

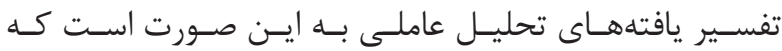

جدول F- نتايج آزمون اثر درون آزمودنى در متغير اختلال استرس بِ از سانحهٔ جانبازان.

\begin{tabular}{|c|c|c|c|c|c|}
\hline اندازة اثر & سطح معنى دارى & مقدار F & درجه آزادى & منبع تغييرات & متغير \\
\hline १८V/. & $\cdots /$ & HEN/D. IQ & سצץ/1 & زمان & \multirow{3}{*}{ اختلال استرس از سانحه } \\
\hline १マ१/. & $\ldots /$ & $1.1 \mathrm{r} / \mathrm{YqV}$ & $f / \cdot \Lambda \Lambda$ & زمان *3روه & \\
\hline- & - & - & NV/TII & خطا (زمان) & \\
\hline
\end{tabular}

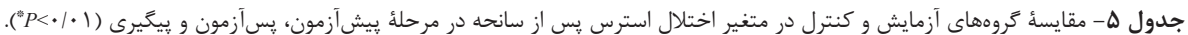

\begin{tabular}{|c|c|c|c|c|c|}
\hline سطع معنى دارى & ثفاوت ميانئين (كروه او ؟r) & $r \circ g$ & $\log$ & 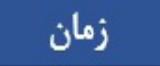 & متغير \\
\hline $1 / \cdots$ & $\cdot / r \Delta \mu$ & TDCS & \multirow{3}{*}{ سرتر الين } & \multirow{12}{*}{ يشي آزمون } & \multirow{12}{*}{ 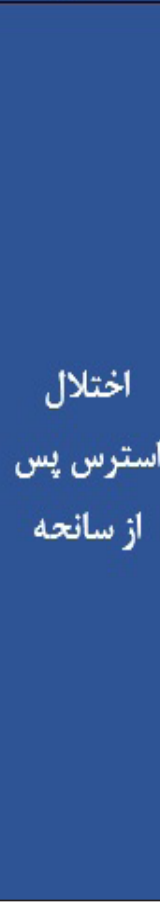 } \\
\hline $1 / \cdots$ & $\cdot|f v|$ & درمان تركيبى & & & \\
\hline $1 / \cdots$ & $\cdot / 111$ & كنترل & & & \\
\hline $1 / \cdots$ & - $/ r \Delta r$ & سرترالين & \multirow{3}{*}{ TDCS } & & \\
\hline $1 / \cdots$ & - INTF & درمان تركيبى & & & \\
\hline $1 / \cdots$ & ט & كنترل & & & \\
\hline $1 / \cdots$ & $\cdot|f V|$ & سرتر الين & \multirow{3}{*}{ درمان تركيبى } & & \\
\hline $1 / \cdots$ & - MTF & TDCS & & & \\
\hline $1 / \cdots$ & $\cdot|\Delta \Lambda|$ & كنترل & & & \\
\hline $1 / \cdots$ & $-\cdot / 111$ & سرترالين & \multirow{3}{*}{ كنترل } & & \\
\hline $1 / \cdots$ & • rma & TDCS & & & \\
\hline $1 / \cdots$ & $-\cdot 1011$ & درمان تركيبى & & & \\
\hline
\end{tabular}




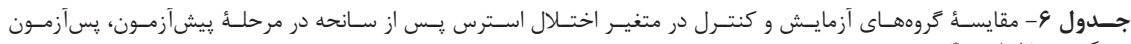

\begin{tabular}{|c|c|c|c|c|c|}
\hline سطح معنى فارى & 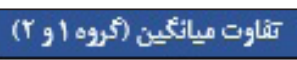 & $r \circ \operatorname{ogg}^{5}$ & $\log f^{5}$ & زمان & هتغير \\
\hline$+/++*$ & $-1+/ 9 F 1^{*}$ & TDCS & \multirow{3}{*}{ سرترالين } & \multirow{12}{*}{ يس آزعون } & \multirow{24}{*}{ 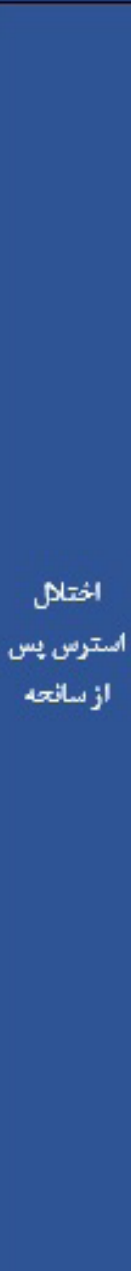 } \\
\hline$+/++*$ & $11 / 1 v \varepsilon^{*}$ & درعان تركيبى & & & \\
\hline$+/++*$ & $-T \Lambda / \Delta \Lambda \Lambda^{*}$ & كنترل & & & \\
\hline$+/++*$ & $1+/ 9 F 1^{*}$ & سرترالين & \multirow{6}{*}{ تركيبع } & & \\
\hline$+/++*$ & $r r / 11 \Lambda^{*}$ & درعان تركيبى & & & \\
\hline$+/+++$ & $-\mid V / F F F V^{*}$ & كنترل & & & \\
\hline$+/++*$ & $-11 / 1 v \varepsilon^{*}$ & سرترالين & & & \\
\hline$+/++*$ & $-r r / 11 \Lambda^{*}$ & TDCS & & & \\
\hline$+/++*$ & $-r q / v q \Delta^{*}$ & كنترل & & & \\
\hline$+/++*$ & $\Gamma \wedge / \Delta A A^{*}$ & سرترالين & \multirow{3}{*}{ كنترل } & & \\
\hline$+/++*$ & $\mid V / \mathscr{F} F V^{*}$ & TDCS & & & \\
\hline$+/++*$ & rq/VGs* & درعان تركيبى & & & \\
\hline $1 /++*$ & $+/ 111$ & TDCS & \multirow{3}{*}{ سرترالين } & \multirow{12}{*}{ بيثيرى } & \\
\hline$+/++*$ & $r V / f 1 r^{*}$ & درمان تركيبى & & & \\
\hline$+/++*$ & $-\| T / F V 1^{*}$ & كنترل & & & \\
\hline $1 /++*$ & $-+/ 111$ & سرترالين & \multirow{3}{*}{ TDCS } & & \\
\hline$+/++*$ & $r V / T q f^{*}$ & درعان تركيبى & & & \\
\hline$+/++*$ & $-1 T / \Delta A A^{*}$ & كنترل & & & \\
\hline$+/++*$ & $-t r / f 1 t^{*}$ & سرترالين & \multirow{3}{*}{ تركيبى } & & \\
\hline$+/++*$ & $-42 / 4 q f^{\prime}$ & TDCS & & & \\
\hline$+/++*$ & $-r M / \wedge \Lambda r^{*}$ & كنترل & & & \\
\hline$+/++*$ & $|r / f V|^{*}$ & سرترالين & \multirow{3}{*}{ كتترل } & & \\
\hline$+/++*$ & $\Pi T / \Delta A \Lambda^{*}$ & TDCS & & & \\
\hline$+1+++*$ & $r q / \wedge \wedge r^{*}$ & درعان تركيبى & & & \\
\hline
\end{tabular}

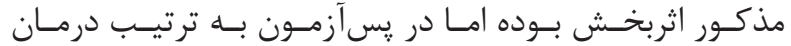

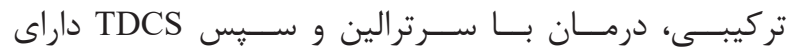

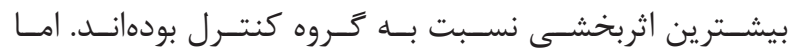

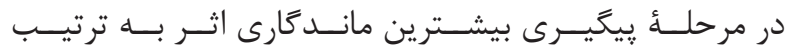

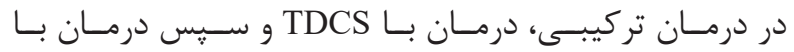

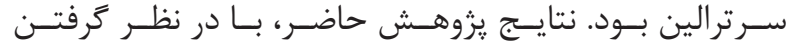

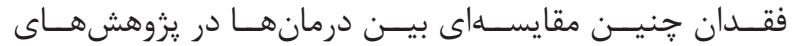

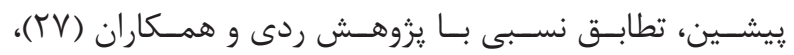

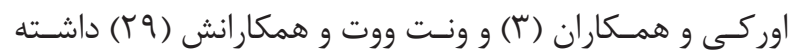

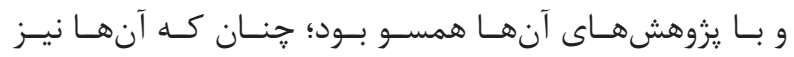

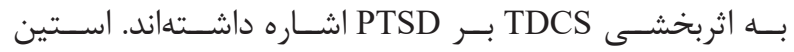

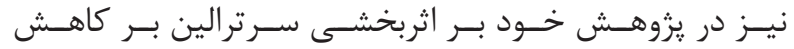

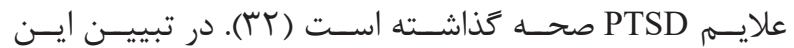

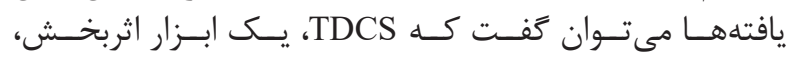

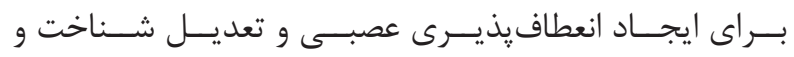

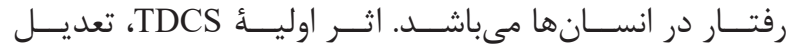

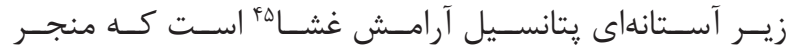

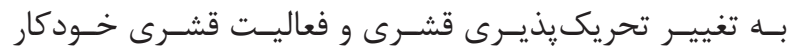

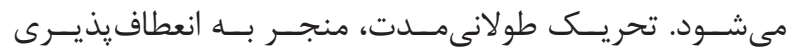

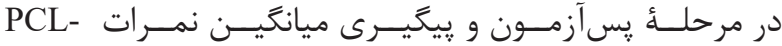

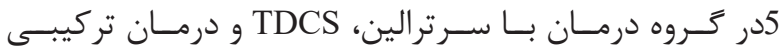

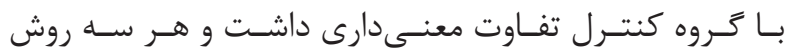

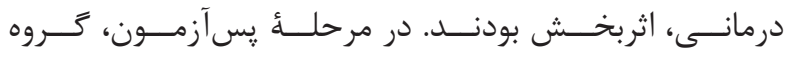

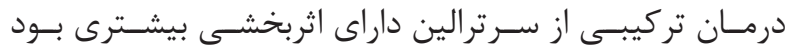

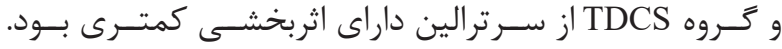

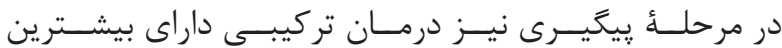

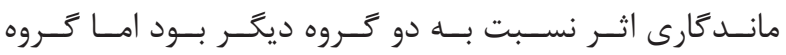

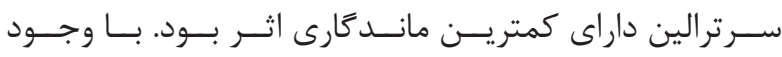

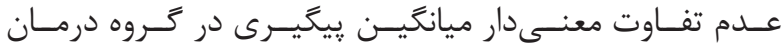

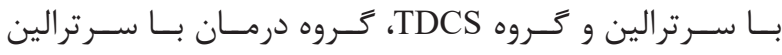

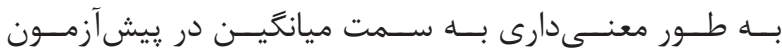

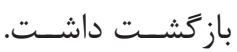

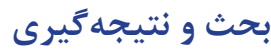

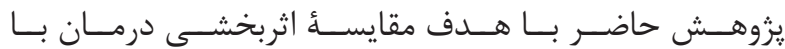

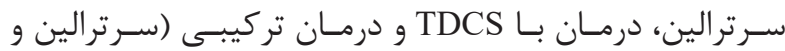

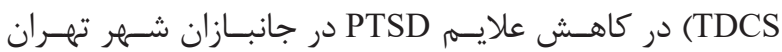

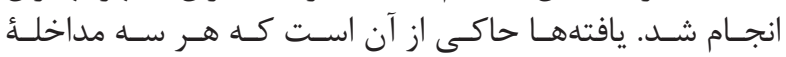




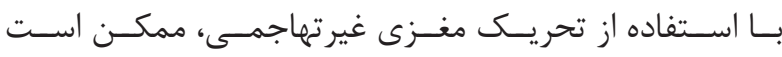

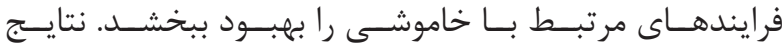

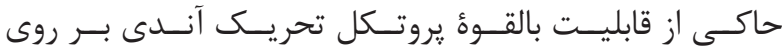

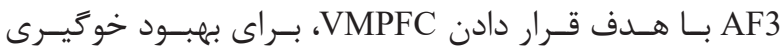

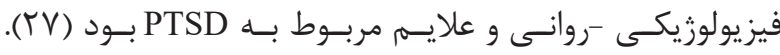

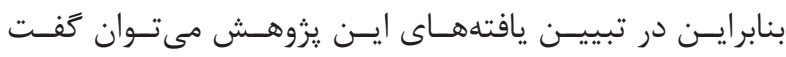

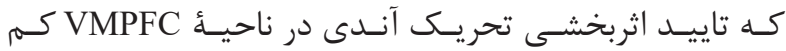

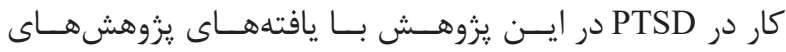

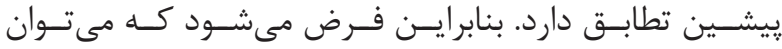

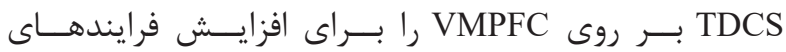

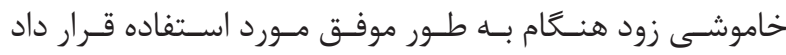

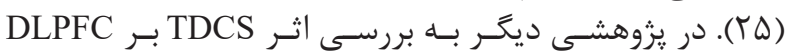

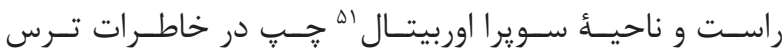

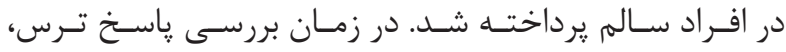

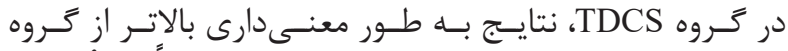

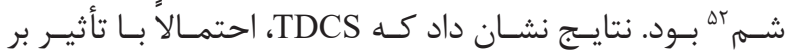

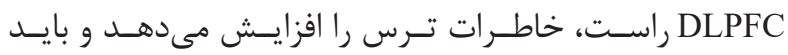

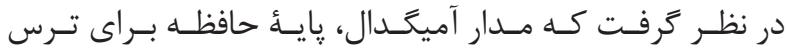

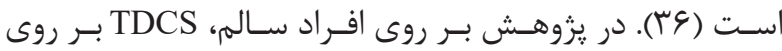
DLPFC (تحريـك آنـدى DLPFC

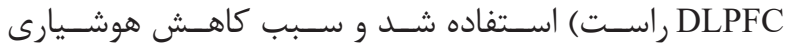

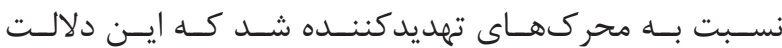

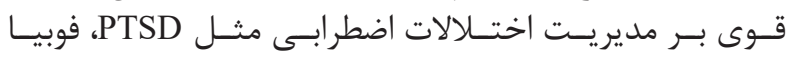

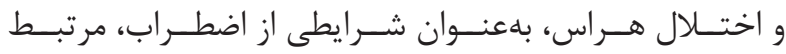

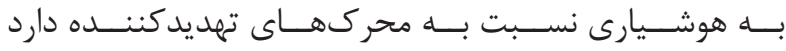

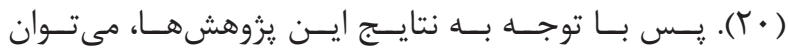

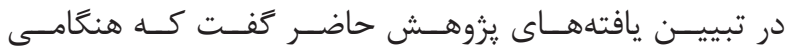

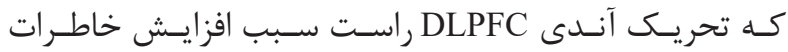

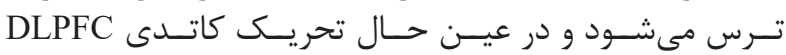

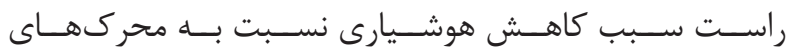

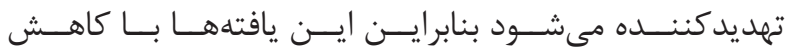

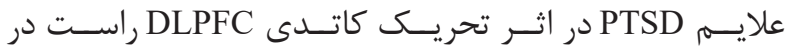
ايسـن ثروهـــ تطابـق دارد.

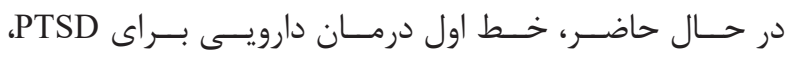

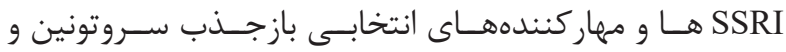

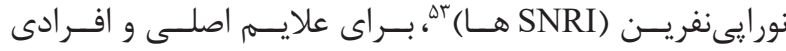

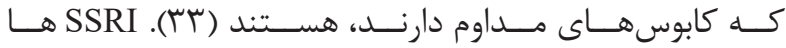

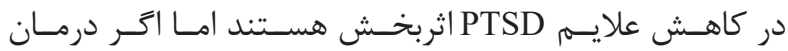

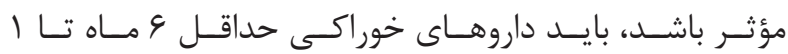

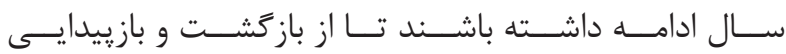

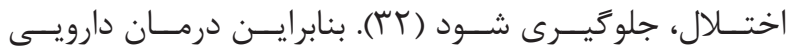

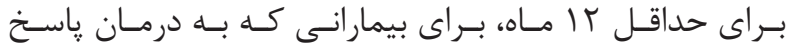

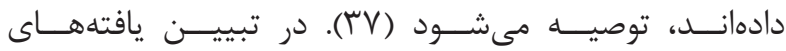

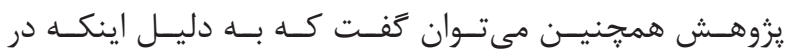

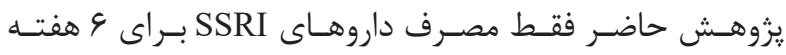

${ }^{46}$ Sub cortical

${ }^{47}$ Amygdala

${ }^{48}$ Hippocampus

${ }^{49}$ Dorsal anterior cingulate cortex

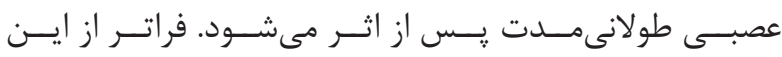

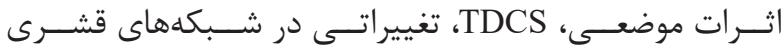

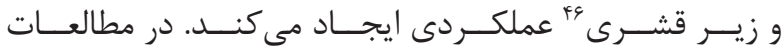

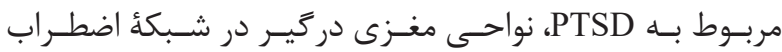

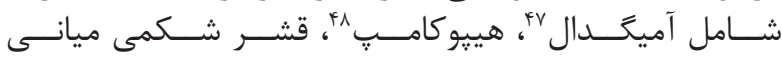

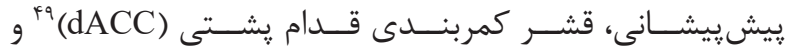

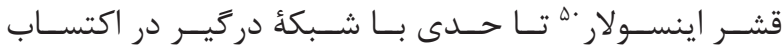

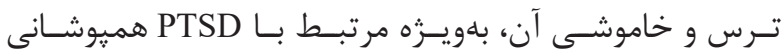

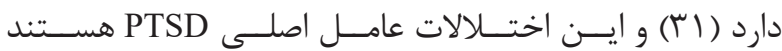

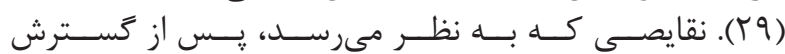

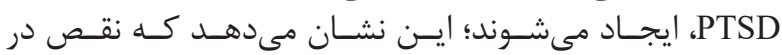

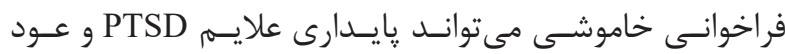

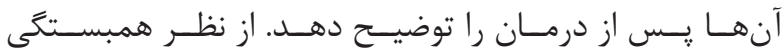

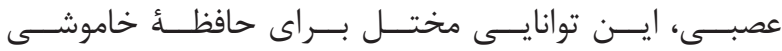

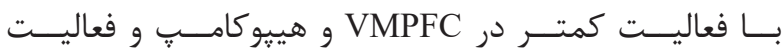

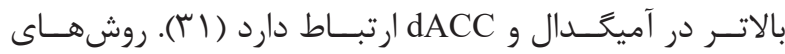

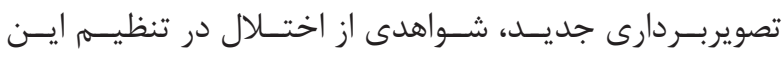

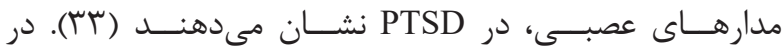

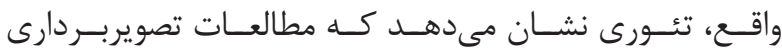

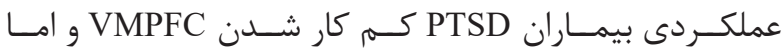

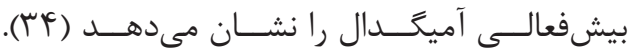

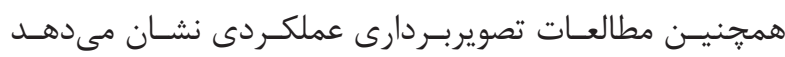

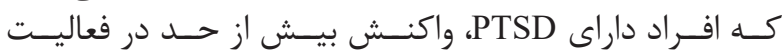

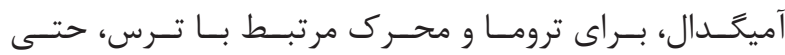

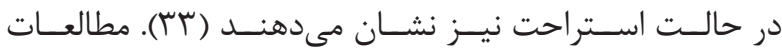

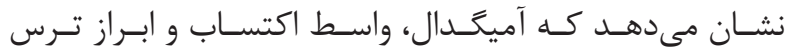

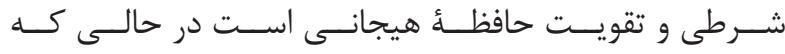

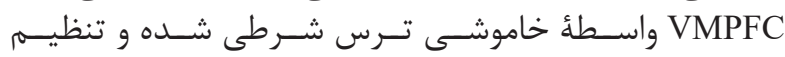

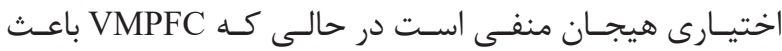

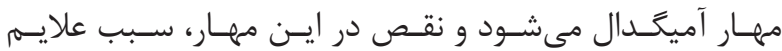

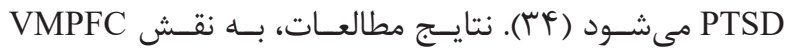

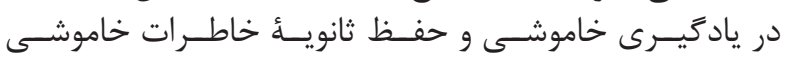

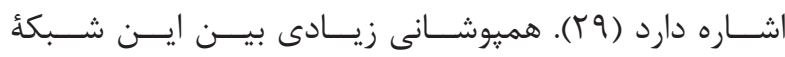

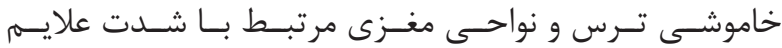

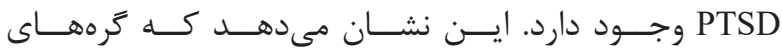

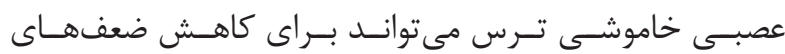

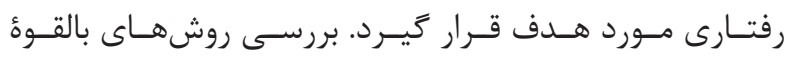

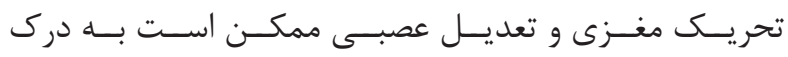

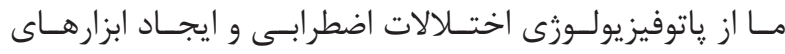

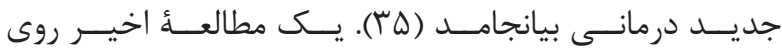

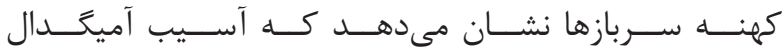

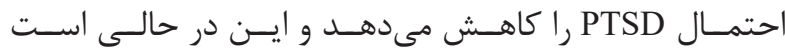

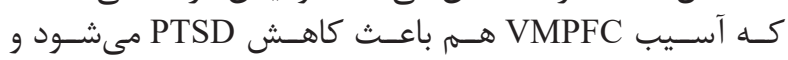

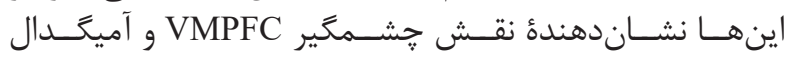

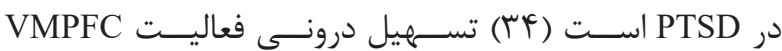

\footnotetext{
${ }^{50}$ Insular cortex

${ }^{51}$ Supraorbital

${ }^{52}$ Sham

${ }^{53}$ Serotonin- norepinephrine reuptake inhibitor
} 


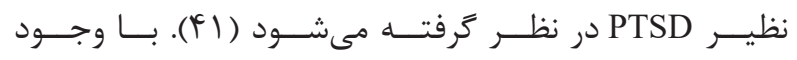

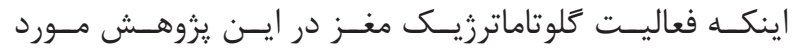

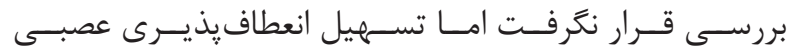

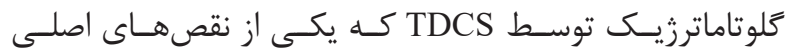

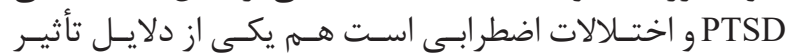

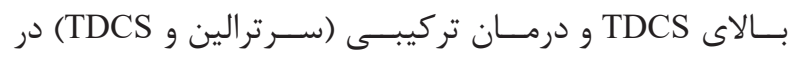

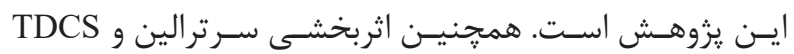

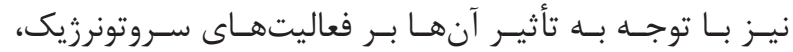

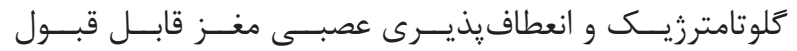

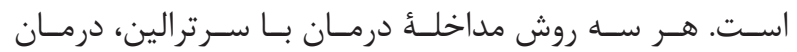

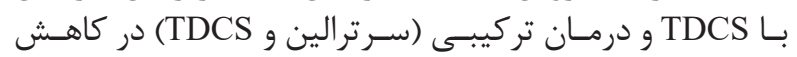

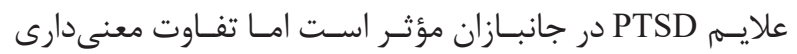

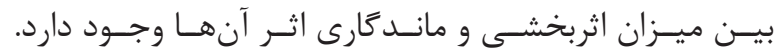

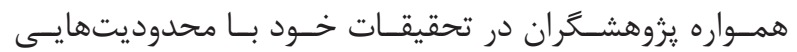

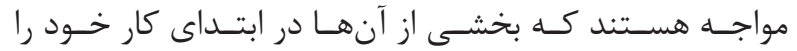

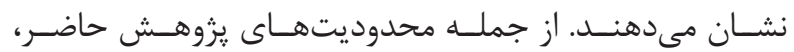

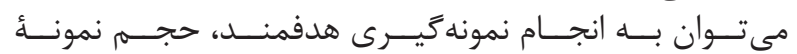

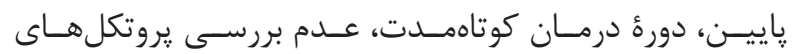

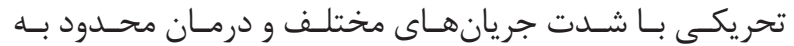

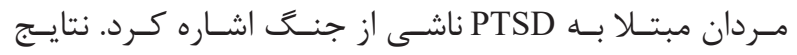

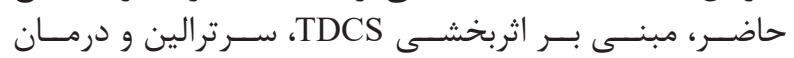

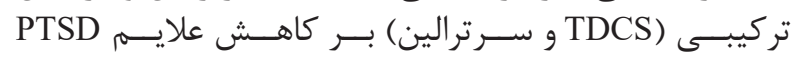

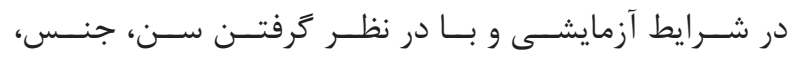

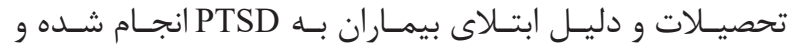

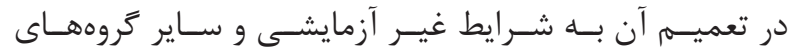

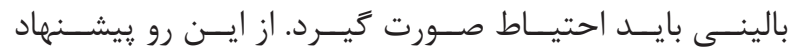

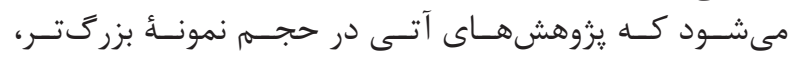

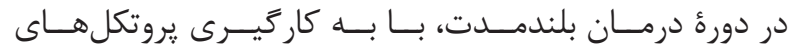

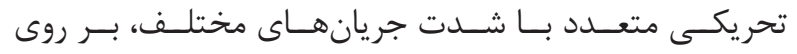

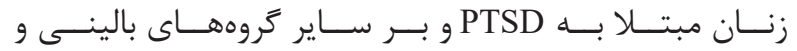

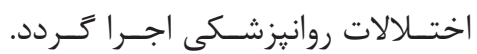

$$
\text { تشكر و قدردانى }
$$

بدينوسـيله از مسـئولان بنيـاد شـهـيد و امــور ايثارگتـران شـهـر

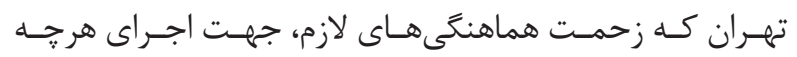

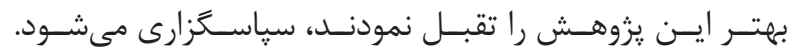

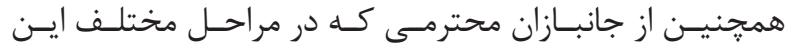

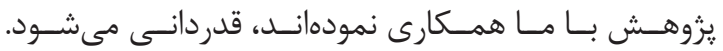

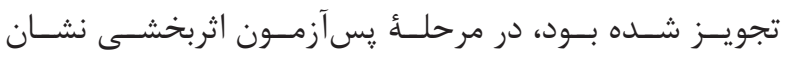

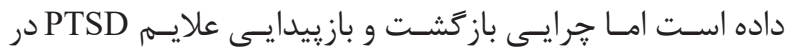

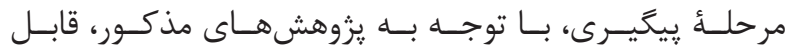

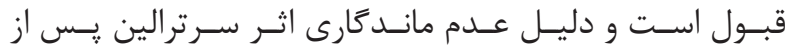

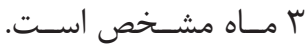

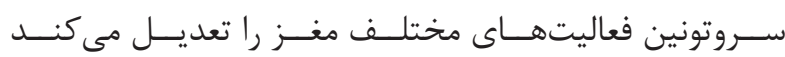

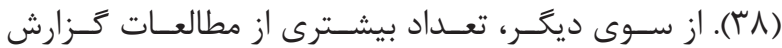

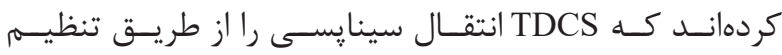

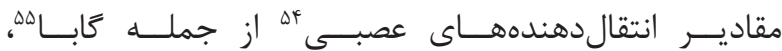

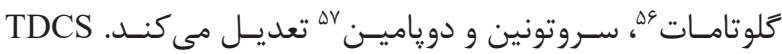

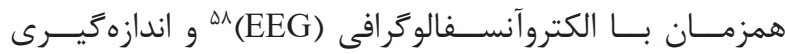

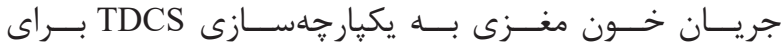

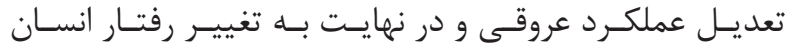

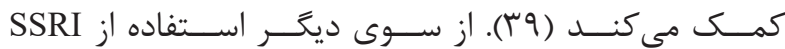

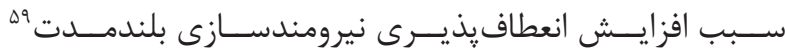

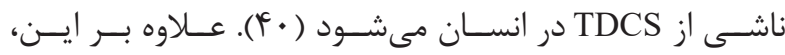

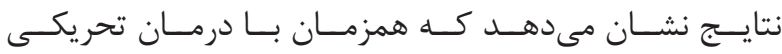

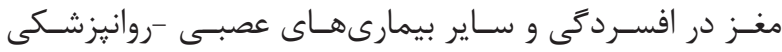

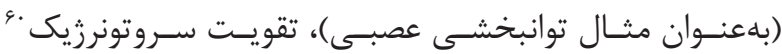

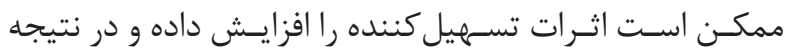

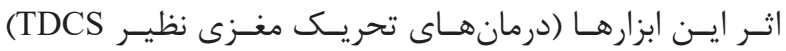

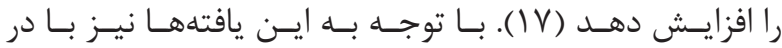

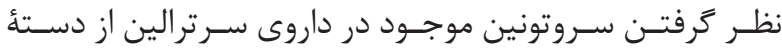

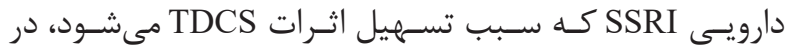

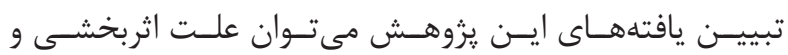

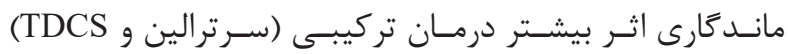

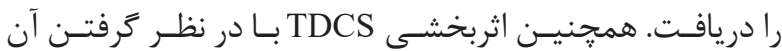

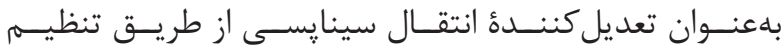

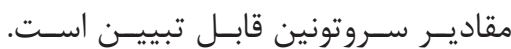

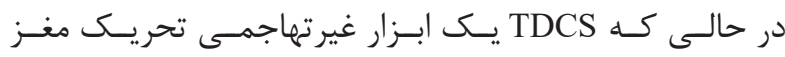

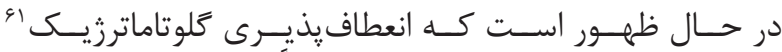

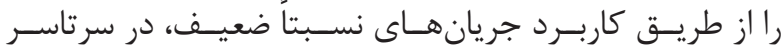

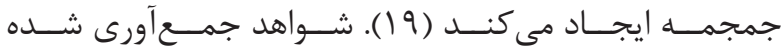

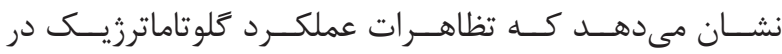

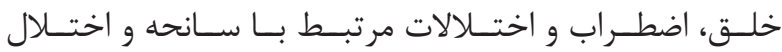

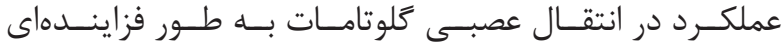

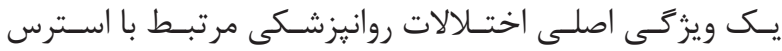

\footnotetext{
${ }^{54}$ Neurotr nsmitters

${ }^{55} \mathrm{GABA}$

${ }^{56}$ Glutamate

${ }^{57}$ Dopamine
}

${ }^{58}$ Electroencephalography

${ }^{59}$ Long term potentiation

${ }^{60}$ Serotonergic

${ }^{61}$ Glutamatergic 
1. Moradi A, Salimi M, Fathi-Ashtiani A. Memory performance among Iranian veterans with posttraumatic stress disorder. Journal of Behavioral Sciences. 2011; 4(4): $269-76$.

2. Pourabaian-Esfahani MH, Esmaeili L, Dabbashi F, Amini M. The effectiveness of music therapy on general health of veterans with post-traumatic stress disorder (P.T.S.D). Knowledge \& Research in Applied Psychology. 2016; 17(4): 41-9.

3. Oraki M, Faraji R, Zarei H, Nejati V. The effectiveness of transcranial direct current stimulation (TDCS) on executive functions of war survivors who suffer from PTSD. Neuropsychology. 2018; 3(4): 103-14.

4. Sadeghi M, Taghva A, Goudarzi N, Rah-Nejat AM. Validity and reliability of persian version of "post -traumatic stress disorder scale" in war veterans. Iranian Journal of War \& Public Health. 2016; 8(4): 243-9.

5. Mandani B, Fakhri A. Study f health related quality of life in posttraumatic stress disorder war veterans. Iranian Journal of War \& Public Health. 2013; 5(2): 18-25.

6. Sadock, BJ, Sadock VA, Ruiz P. Kaplan \& shaddock's synopsis of psychiatry. Behavioral Science. $11^{\text {th }}$ ed. Tehran. Arjmand Press. 2015; P. 783-7.

7. Shalev A, Liberzon I, Marmar C. Post- traumatic stress disorder. N Engl J med. 2017; 376(25): 2459-69.

8. Kelmendi B, Adams TG, Yarnell S, Southwick S, Abdallah CG, Krystal JH. PTSD: from neurobiology to pharmacological treatments. Eur J Psychotraumatol. 2016; 7(2): 318-28.

9. Khodaie-Ardakani M, Khanjani M, Mirabzadeh A, Bahmani B, Abtahi E, Sadighi G, et al. Effect of exposure to the traumatic event location in war veterans with post-traumatic stress disorder. Iranian Journal of War and Public Health. 2014; 6(2): 10-7.

10. Puetz TW, Youngstedt, SD, Herring MP. Effects of pharmacotherapy on combat- related PTSD, anxiety and depression: a systematic review and meta- regression analysis. PloS One. 2015; 10(5): 652-9.

11. Asnis GM, Kohn SR, Henderson M, Brown NL. SSRI's versus non-SSRI's in post-traumatic stress disorder. Drugs. 2004; 64(4): 383-404.

12. MacNamara A., Rabinak CA, Kennedy AE, Fitzgerald DA, Liberzon I, Stein MB, et al. Emotion regulatory brain function and SSRI treatment in
PTSD: neural correlates and predictors of change. Neuropsychopharmacology. 2016; 41(2): 611-8.

13. Julien RM, Advokat CD, Comaty JE. Antidepressant. A primer of drug action: comprehensive guide to the actions, uses, and side effects of psychoactive drugs. $12^{\text {th }}$ ed. Tehran. Arjmand Press. 2015; P. 179-80.

14. Nikvarz N, Khalili H, Sahraie Z, Ghaeli P. Pharmacotherapy of neuropsychiatric diseases. $1^{\text {st }}$ ed. Tehran. Arjmand Press. 2013; P. 21-5.

15. Azar M, Nohi S. Common psychiatric drugs. $2^{\text {nd }}$ ed. Tehran. Arjmand Press. 2010; P. 59-62.

16. Kuo MF, Chen PS, Nitsche MA. The application of tDCS for the treatment of psychiatric diseases. Int Rev Psychiatry. 2017; 29(2): 146-67.

17. Nitsche MA, Kou MF, Karrasch R, Wachter B, Liebetanz D, Paulus W. Serotonin affects transcranial direct current-induced neuroplasticity in humans. Biol Psychiatry. 2009; 66(5): 503-8.

18. Davidson J. Vintage treatments for PTSD: a reconsideration of tricyclic drugs. J Psychopharmacol. 2015 ; 29(3): 264-9.

19. Kuo Mf, Paulus W, Nitsche MA. Therapeutic effects of non-invasive brain stimulation with direct currents (tDCS) in neuropsychiatric diseases. Neuroimage. 2014; 85: $948-60$

20. Kar SK, Sarkar S. Neuro-stimulation techniques for the management of anxiety disorders: an update. Clin Psychopharmacol Neurosci. 2016; 14(4): 330-7.

21. Kim JW, Lee, J. Application of transcranial direct current stimulation in psychiatry. J Korean Neuropsychiatr Assoc. 2016; 55(3): 158-67.

22. Arkan A, Yaryari F. The effect of transcranial direct current stimulation (TDCS) on the working memory in healthy people. Journal of Cognitive Psychology. 2014; 2(2): 10-7.

23. Ebadi M, Hosseini F, Pahlevan F, EsmaeeilzadehAkhoundi M, Farhadi V, Asqari R. The effectiveness of transcranial direct current stimulation (tdcs) on working memory in patients with major depression. Arak Medical University Journal. 2017; 20(122): 38-47.

24. Vafaye- Sisakht S, Ramezani K. The effects of transcranial direct current stimulation on mental health of veterans with psychiatric disorders. Shefaye Khatam. 
2017; 5(2): 36-42.

25. Dittert N, Huettner S, Polak T, Herrmann MJ. Augmentation of fear extinction by transcranial direct current stimulation (TDCS). Front Behav Neurosci. 2018; 12: 76-82.

26. Hampstead BM, Garcia S, Schlaefflin S, Porter KE, Smith ER, Martis B, et al. HD- TDCS for combat related PTSD: a case series. Brain Stimul. 2017; 10(2): 514-22.

27. Van't Wout-Frank M, Reddy M, Greenberg B, Philip N. Combining transcranial direct current stimulation with virtual reality exposure for PTSD. Biological Psychiatry. 2017; 81(10): 258-66.

28. Van't Wout- Frank M, Shea T, Larson VC, Greenberg BD, Philip NS. Combined transcranial direct current stimulation with virtual reality exposure for posttraumatic stress disorder: Feasibility and pilot results. Brain Stimul. 2019; 12(1): 41-3.

29. Van't Wout M, Longo SM, Reddy MK, Philip NS, Bowker MT, Greenberg BD. Transcranial direct current stimulation may modulate extinction memory in posttraumatic stress disorder. Brain Behav. 2017; 7(5): 681-92.

30. Delavar, A. Theoretical and practical research in the humanities and social sciences. $9^{\text {th }}$ ed. Tehran. Roshd Press. 2011; P. 315-23.

31. Brunoni A, Nitsche M, Loo C. Transcranial direct current stimulation in neuropsychiatric disorders. $1^{\text {st }}$ ed. Switzerland: Springer International Publishing. 2016.

32. Stein MB. Pharmacotherapy for post-traumatic stress disorder in adults. (2015); https://www.uptodate.com/ contents.

33. BakerDG, Lerman I, Espejo EP, McLay R. Treatments for Post-Traumatic stress disorder: pharmaceutical and electrophysiologic considerations. Current Treatment Options in Psychiatry. 2015; 2(1): 73-85.

34. Koenigs M, Grafman J. Posttraumatic stress disorder: the role of medial prefrontal cortex and amygdala. The Neuroscientist. 2009; 15(5): 540-8.

35. Marin MF, Camprodon JA, Dougherty DD, Milad MR. Device-based brain stimulation to augment fear extinction: Implications for PTSD treatment and beyond. Depression and Anxiety. 2014; 31(4): 269-78.

36. Mungee A, Kasser P, Feeser M, Nitsche, MA, Schiller D, Bajbouj M. Transcranial direct current stimulation of the prefrontal cortex: a means to modulate fear memories. NeuroReport. 2014; 25(7): 480-4.

37. Aslan EA. Up-to-date psychopharmacological treatments in post-traumatic stress disorder. Klinik Psikofarmakoloji Bulteni. 2018; 28: 332-9.

38. Batsikadze G, Paulus W, Kou MF, Nitsche MA. Effect of serotonin on paired associative stimulationinduced plasticity in the human motor cortex. Neuropsychopharmacology. 2013; 38(11): 2260-7.

39. Pulagar VM. Direct electric stimulation to increase cerebrovascular function. Front Syst Neurosci. 2015; 9: 54-62.

40. Kuo HI, Paulus W, Batsikadze G, Jamil A, Kuo MF, Nitsche MA. Chronic enhancement of serotonin facilitates excitatory transcranial direct current stimulation-induced neuroplasticity. Neuropsychopharmacology. 2016; 41: 1223-30.

41. Averill LA, Purohit P, Averill CL, Boesl MA, Krystal $\mathrm{JH}$, Abdallah CG. Glutamate dysregulation and glutamatergic therapeutics for PTSD: Evidence from human studies. Neurosci Lett. 2017; 649: 147-55. 